\title{
Long-Span Program Behavior Modeling and Attack Detection
}

\author{
XIAOKUI SHU, IBM Research \\ DANFENG (DAPHNE) YAO, Virginia Tech \\ NAREN RAMAKRISHNAN, Virginia Tech \\ TRENT JAEGER, Pennsylvania State University
}

\begin{abstract}
Intertwined developments between program attacks and defenses witness the evolution of program anomaly detection methods. Emerging categories of program attacks, e.g., non-control data attacks and data-oriented programming, are able to comply with normal trace patterns at local views. This article points out the deficiency of existing program anomaly detection models against new attacks and presents long-span behavior anomaly detection (LAD), a model based on mildly context-sensitive grammar verification. The key feature of $\mathrm{LAD}$ is its reasoning of correlations among arbitrary events that occurred in long program traces. It extends existing correlation analysis between events at a stack snapshot, e.g., paired call and ret, to correlation analysis among events that historically occurred during the execution. The proposed method leverages specialized machine learning techniques to probe normal program behavior boundaries in vast high-dimensional detection space. Its two-stage modeling/detection design analyzes event correlation at both binary and quantitative levels. Our prototype successfully detects all reproduced real-world attacks against sshd, libpcre, and sendmail. The detection procedure incurs $0.1 \mathrm{~ms}$ to $1.3 \mathrm{~ms}$ overhead to profile and analyze a single behavior instance that consists of tens of thousands of function call or system call events.
\end{abstract}

CCS Concepts: • Security and privacy $\rightarrow$ Intrusion detection systems; Formal methods and theory of security; Systems security;

Additional Key Words and Pharses: Intrusion detection, program analysis, anomaly detection, contextsensitive grammar, co-occurrence analysis, event frequency correlation, machine learning

\section{ACM Reference format:}

Xiaokui Shu, Danfeng (Daphne) Yao, Naren Ramakrishnan, and Trent Jaeger. 2017. Long-Span Program Behavior Modeling and Attack Detection. ACM Trans. Priv. Secur. 20, 4, Article 12 (September 2017), 28 pages. https://doi.org/10.1145/3105761

This work has been supported by ONR grant N00014-13-1-0016, AFRL and DARPA grant FA8650-15-C-7561, ARO YIP W911NF-14-1-0535, ARL W911NF-13-2-0045, and Army Research Laboratory W911NF-13-2-0045 (ARL Cyber Security CRA). The views and conclusions contained in this document are those of the authors and should not be interpreted as representing the official policies, either expressed or implied, of the Army Research Laboratory or the U.S. Government. The U.S. Government is authorized to reproduce and distribute reprints for Government purposes notwithstanding any copyright notation here on.

A preliminary version of the work appeared in the Proceedings of the 2015 ACM Conference on Computer and Communications Security (CCS) [53].

Authors' addresses: X. Shu, 1101 Kitchawan Rd, Yorktown Heights, NY 10598; email: xiaokui.shu@ibm.com; D. (Daphne) Yao, 2202 Kraft Drive, Blacksburg, VA 24060; email: danfeng@vt.edu; N. Ramakrishnan, VTRC-Arlington, Room 5-026, 900 North Glebe Road, Arlington, VA 22203; email: naren@cs.vt.edu; T. Jaeger, 346A IST Building, University Park, PA 16802; email: tjaeger@cse.psu.edu.

Permission to make digital or hard copies of all or part of this work for personal or classroom use is granted without fee provided that copies are not made or distributed for profit or commercial advantage and that copies bear this notice and the full citation on the first page. Copyrights for components of this work owned by others than the author(s) must be honored. Abstracting with credit is permitted. To copy otherwise, or republish, to post on servers or to redistribute to lists, requires prior specific permission and/or a fee. Request permissions from permissions@acm.org.

2017 Copyright is held by the owner/author(s). Publication rights licensed to ACM.

ACM 2471-2566/2017/09-ART12 \$15.00

https://doi.org/10.1145/3105761 


\section{INTRODUCTION}

Attacks to programs are one of the oldest and fundamental threats to computing systems, which evolve and constitute latest attack vectors and advanced persistent threats. Anomaly-based intrusion detection discovers aberrant executions caused by attacks, misconfigurations, program bugs, and unusual usage patterns. The approach models normal program behaviors instead of the threats. It does not bear time lags between emerging attacks and deployed countermeasures as standard defenses do, which are built upon retrospects of inspected attacks. The merit of program anomaly detection is its independence from attack signatures. This property potentially enables proactive defenses against new and unknown threats.

The detection accuracy of program anomaly detection methods relies on the precision of normal program behavior description and the completeness of the training [54]. Primitive program attacks, e.g., return addresses manipulation and library/system call injection, result in great variation from normal behaviors. Thus, they can be distinguished from relatively imprecise descriptions of normal program behaviors, e.g., $n$-gram system call anomaly detection [12].

Program anomaly detection models evolve as attacks share more and more similarities with normal behaviors. Attacks using explicit control flow manipulation, e.g., tampering with return addresses on the stack, are no longer effective due to the deployment of standard defense mechanisms such as non-executable stack, address space layout randomization, and control-flow integrity. New program attacks utilize indirect means of control flow manipulation, e.g., data-oriented programming [25], or abuse programs within legal control flows, e.g., denial of service attacks. The emerging stealthy attacks diminish the effectiveness of existing anomaly-based intrusion detection models to distinguish them from normal program executions.

Take the remote authentication subroutine in sshd as an example. An attacker can launch a stealthy attack by overwriting flag variable recording authentication results in the authentication subroutine. The overwriting prior to specific authentication operations enables the attacker to bypass critical security control and log in after a failed authentication. There is no illegal control flow or short abnormal system call sequences in this attack. Therefore, it is difficult for existing models to detect. The sshd attack involves breaches of data integrity for altering the execution path. However, this is not necessary. Denial of service attacks and some data retrieval attacks leverage legal-but-unusual execution patterns to fulfill effective attacks. Such attacks can only be detected by existing anomaly detection methods to some extent.

Program anomaly detection utilizes execution traces to describe program behaviors and search for anomalous behaviors [52]. They can be abstracted as former grammar parsers to validate program traces, and existing models are restricted to regular grammar parsers $[11,12,28]$ and contextfree grammar parsers $[10,19,30,49]$. Some comprehensive approaches extend deterministic language parsers to probabilistic ones [15, 22, 36, 63], verify some additional data-flow information [3, $18,19,38]$, or perform simple frequency analysis [14, 15, 61]. However, none of them is designed to represent a context-sensitive grammar parser, which is able to better recognize normal program behaviors and detect aforementioned attacks.

The key difference between a context-sensitive grammar model and existing (regular or contextfree grammar) models is the ability to correlate arbitrary events in a long program trace. Two segments of code executed during a run may correlate by specific control or data links. Contextsensitive grammar parsing enables the mining of event correlations in long program traces. The correlations reveal specific behavior properties of normal executions, which could distinguish them from anomalous runs and attacks. For instance, the aforementioned sshd attack breaks the correlation between the authentication result and the login operation.

The complete context-sensitive analysis is impractical to reach by a real-world detection tool due to non-polynomial training complexity [54]. However, it is possible to construct parsers that 
recognize context-sensitive properties in the traces yet avoid the impractical complexity of complete context-sensitive grammars.

We present long-span behavior anomaly detection (LAD)-a mildly context-sensitive grammar program anomaly detection model to characterize program behaviors and detect stealthy attacks. We approach the anomaly detection problem from the machine learning perspective and blueprint a two-stage data mining solution for event correlation analysis in long program traces. LAD can be abstracted as a stochastic, mildly context-sensitive language parser. It consists of a training phase (the stochastic language construction procedure) and a detection phase (new behavior/trace testing). The context-sensitive property enables the model to learn correlations between events that are millions of events away in the trace. The stochastic language estimates proper boundaries of normal behaviors in a high-dimensional detection space and mitigates potential oversensitive detection due to insufficient training samples.

Our approach overcomes two major challenges in constructing a context-sensitive grammar model for program anomaly detection.

Training scalability challenge: the complete event reasoning in extremely long trace segments forms a vast detection space, the size of which is exponential to the length of the training trace segments. It requires exponential training time to converge. Insufficient training results in high false-positive rates as tested in many $n$-gram methods with $n>40$ for long segment analysis. Our approach reduces the potential exponential-size space to a constant-size high-dimensional detection space by profiling program behavior instances into fixed size matrices. The matrix profiles are initialized by static program analysis and filled by program behavior instances (dynamic traces).

Behavior diversity challenge: various functionalities in real-world programs lead to diverse program behaviors. Diverse normal data points in the high-dimensional detection space make it difficult to seek a hyperplane in traditional classifiers, e.g., one-class SVM, for precisely distinguishing anomalous behaviors from normal ones. Stealthy attacks can often exploit the imprecision of normal boundaries generated by traditional classifiers and subvert the detection. Our two-stage design recognizes diverse normal behaviors in clusters and performs precise characterizations of normal behaviors inside each cluster.

The contributions of our work are summarized as follows.

- We identify the need to correlate events in long program traces for the detection of stealthy program attacks that alter execution paths instead of control flows. We formalize a stochastic mildly context-sensitive language model for mining arbitrary event correlations in long program traces and estimating normal boundaries in a high-dimensional detection space.

- We embody the proposed mildly context-sensitive language model with a two-stage data mining approach, which consists of a constrained agglomerative clustering algorithm for addressing the behavior diversity challenge and a combination of probabilistic and deterministic models for precise intra-cluster behavior modeling. The two-stage detection approach mines event correlations from fixed-size matrix profiles of program behaviors, which addresses the training scalability challenge.

-We prototype our program anomaly detection approach on Linux and evaluate its detection capability, accuracy, and performance with sshd, libpcre, and sendmail. Our prototype is trained with over 22,000 normal profiles and detect over 800 reproduced real-world attacks. High detection accuracy is demonstrated against four categories of synthetic anomalies, and the testing of a single program behavior profile with $1 \mathrm{k}$ to $50 \mathrm{k}$ function/system call events incurs only $0.1 \mathrm{~ms}$ to $1.3 \mathrm{~ms}$. 


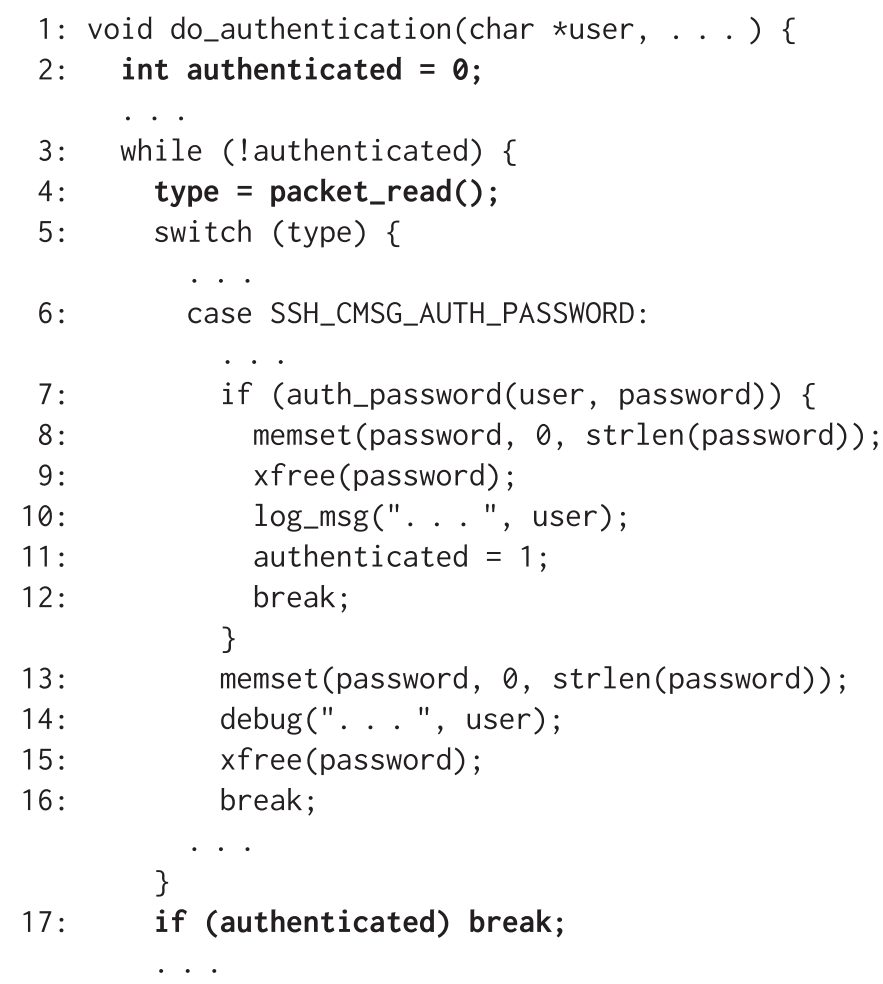

Fig. 1. sshd flag variable overwritten attack.

\section{SECURITY MODEL}

We discuss stealthy attacks that do not directly alter control flows, point out the need of event correlation analysis in long program traces, and present a mildly context-sensitive grammar parser for the detection of such attacks.

\subsection{Aberrant Path Attack}

We study a category of stealthy attacks - aberrant path attacks, which contain infeasible, inconsistent, or aberrant execution paths, but they obey legitimate control-flow graphs. Aberrant path attacks can evade existing detection mechanisms because of the following properties of the attacks:

- not conflicting with control-flow graphs

- not incurring anomalous call arguments

- not introducing unknown short call sequences

Aberrant path attacks are realistic threats and gain in popularity since primitive attacks are less effective due to memory protection, e.g., address space layout randomization (ASLR) [50]. A popular example is the sshd flag variable overwritten attack first described by Chen et al. [6]. The attack takes advantage of an integer overflow vulnerability found in several implementations of the SSH1 protocol [35]. Illustrated in Figure 1, an attacker can overwrite the flag integer authenticated when the vulnerable procedure packet_read() is called. If authenticated is overwritten to a non-zero value, line 17 is always True and auth_password() on line 7 is no longer effective. 
Aberrant path attacks do not only allow the attacker to bypass authentication procedures, but can also be leveraged to cover Turing-complete operations as control flow manipulation attacks. We list four common types of aberrant path attacks as follows.

(1) Non-control data attacks that reorganize existing control flows to create new execution paths fall into the categories of aberrant path attacks. ${ }^{1}$ The attacks hijack programs without manipulating their control data (data loaded into program counter, e.g., return addresses). It is named by $\mathrm{Chen}$ et al. [6] and generalized by $\mathrm{Hu}$ as data-oriented programming [24, 25]. Hu utilizes compromised variables to construct loop trampolines and achieve Turing-complete functionalities without directly tampering control data.

(2) Workflow violation attacks exploit weak workflow enforcements of a system. The attacker usually executes part of the program to bypass access control [7], leak critical information, or disable a service (e.g., trigger a deadlock). One example is presentation layer access control bypass in web applications. If the authentication is only enforced at the presentation layer, which is not securely coupled to its underlying business logic layer, an attacker can directly access the business logic layer and read/write data.

(3) Exploit preparation is a common step preceding the launch of an exploit payload. It usually utilizes legal control flows to load essential libraries, arranges memory space (e.g., heap feng shui [55]), seeks addresses of useful code and data fragments (e.g., ASLR probing [50]), and/or triggers particular race conditions.

(4) Service abuse attacks do not take control of a program. Instead, the attacks utilize legal control flows to compromise the availability (e.g., Denial of Service attack), confidentiality (e.g., Heartbleed data leak [23]), and financial interest (e.g., click fraud) of target services.

\subsection{Security Goal: Event Correlation Mining in Long Program Traces}

The key to the detection of aberrant path attacks is the knowledge of instruction correlations in a long program trace. Even legal control flows are preserved; the execution paths of the program under attacks are different than normal. Therefore, the attacker can achieve their attack goals.

Taking the sshd flag variable overwritten attack (Figure 1) as an example, normal execution paths should contain lines 8-12 if line 17 is True (a shell is spawned afterward). The attack trace, on the contrary, contains the shell spawn procedure (line 18-) without the execution of lines 8-12. Co-occurrence between instructions in an execution can be analyzed to detect such an attack.

Another example is the loop trampoline proposed by Hu to construct a Turing-complete noncontrol data attack [25]. In this attack, a vulnerable variable in a loop branch is exploited to prepare a gadget $-\mathrm{a}$ fraction of the malicious payload. The attacker executes the loop trampoline at an unusual pattern to chain a string of gadgets together and achieve Turing-complete operations. The attack reflects an unusual pattern of the loop usage because of the gadget construction. It could also result in broken co-occurrence among instructions in normal runs.

The goal of the detection is to learn event correlations in long program traces. Events refer to function calls, jumps, or even generic instructions in a program trace. The correlations append additional information to control flows, making it possible to enforce co-occurrences of branches as well as different portions of a process.

We define event correlation in two levels.

\footnotetext{
${ }^{1}$ Simple non-control data attacks that only exploit arguments of legitimate exec system calls may not result in explicit aberrant path attacks.
} 
(1) Event co-occurrence denotes the binary relation between co-occurred events in a long program trace. Repeating events should be deduplicated, and event appearances are studied in binary forms.

(2) Event occurrence frequency relation denotes quantitative relation among event occurrence frequencies in a long program trace. It provides more fine-grained knowledge about the execution path than event co-occurrences.

\subsection{Security Model: A Stochastic Mildly Context-Sensitive Language}

We first define a program trace as the building block to formalize program anomaly detection models. Then we present a mildly context-sensitive language model for characterizing event cooccurrences and event frequency relations in a long program trace.

Definition 2.1. A program trace $T$ is the sequence of all instructions executed in an autonomous portion of a program execution.

$T$ records the sequence of all executed instructions (addresses and arguments). In real-world detection systems, select instructions are used to represent all instructions in the construction of $T$. The simplification sacrifices precision for practicality, e.g., smaller tracing and modeling overhead. For example, $T$ only contains system call instructions, e.g., SYSENTER, in system call anomaly detection systems.

To build practical detection systems, $T$ can be a long portion of a finite or infinite ${ }^{2}$ program trace. In practice, $T$ is partitioned from an entire trace based on boundaries of program functionalities to represent program behaviors with semantic meanings. Examples of partitioning for security analysis include (i) critical subroutine call/return, (ii) threads creation/termination, or (iii) the entire execution of a small program.

Given a set of normal program traces (generated from dynamic or static/synthetic program analysis), a program anomaly detection system forms a formal grammar to accept all normal traces and reject others. The detection system can be written as the set of all normal program traces, i.e., a formal language: $L=\{T \mid T$ is normal $\}$.

To fulfill the security goal of describing arbitrary event correlations in long program traces, one needs a context-sensitive language model to parse program traces.

Theorem 2.2. A program anomaly detection model describing arbitrary event correlations in a long program trace is a context-sensitive language model.

Proof. We prove the contrapositive of Theorem 2.2: a context-free language cannot model arbitrary event correlation in a long program trace.

Figure 2 illustrates a simple block of code that results in a strong relation of the occurrence frequencies of system calls $s_{1}, s_{2}$, and $s_{3}$ in an execution trace. The traces of the program can be described as a language $\dot{L}=\left\{\cdots s_{1}^{n} s_{2}^{n} s_{3}^{n} \cdots\right\}=\left\{\cdots s_{1} s_{2} s_{3} \cdots, \cdots s_{1} s_{1} s_{2} s_{2} s_{3} s_{3} \cdots, \ldots\right\}$. It is proved that the formal language $\ddot{L}=\left\{s_{1}^{n} s_{2}^{n} s_{3}^{n}\right\}$ is not a context-free language- $\ddot{L}$ does not satisfy the pumping lemma property shared among all context-free languages [2]. Therefore, $\dot{L}$ (a superset of $\ddot{L}$ ) is not a context-free language.

Long program traces modeled as strings establish a high-dimensional space for modeling, which makes the training of a generic context-sensitive language model impractical. When processed as strings, each position in the traces, e.g., the $i^{\text {th }}$ item, establishes one dimension in the detection space. The dimensionality of the space equals to the length of the longest trace.

\footnotetext{
${ }^{2} \mathrm{~A}$ program could run continuously and yield an infinite trace.
} 


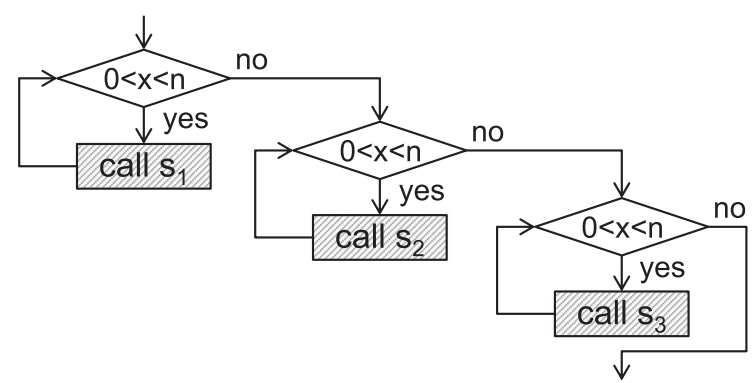

Fig. 2. An example of event relation restricted by induction variables.

Table 1. An Example of $\tilde{L}$

\begin{tabular}{|c|c|c|}
\hline \multicolumn{2}{|l|}{$\frac{\sum=\left\{s_{1}, s_{2}, s_{3}\right\}}{T \Gamma^{*}}$} & program behavior modeled by three system calls \\
\hline \multicolumn{2}{|l|}{$T \in \Sigma^{*}$} & a trace is an arbitrary combination of items in $\Sigma$ \\
\hline \multicolumn{2}{|l|}{$\underline{f_{i}=\text { T.count }\left(s_{i}\right) \mid 1 \leq i \leq 3}$} & $f_{i}$ represents the frequency of $s_{i}$ in $T$ \\
\hline$\left\{\begin{array}{l}0 \\
\min \left(f_{3} / f_{1}, 1\right)\end{array}\right.$ & $\begin{array}{l}f_{1} \neq f_{2} \\
f_{1}=f_{2}\end{array}$ & probability generation \\
\hline$\eta=0.5$ & & ring probability threshold \\
\hline
\end{tabular}

The detection system in this example accepts program traces with equal numbers of $s_{1}$ and $s_{2}$ and some $s_{3}$ that occur more than half the time of $s_{1}$.

To build a practical detection mechanism, we modify the generic context-sensitive language to avoid exponential training convergence time and oversensitive detection caused by sampled normal behaviors in training. The former refers to the exponential number of traces/objects to model regarding the length of the traces. The latter refers to false positives caused by unsampled normal behaviors/traces that are recognized incorrectly by the detection system.

- The key to our non-exponential time solution is to focus on the event relational information other than the order information among events in traces. We build a restricted contextsensitive language that only characterizes the relation quantitatively among events, but not the order of events in long traces. Our language is a generalization of the context-sensitive language Bach [47]. The latter only characterizes strings with equally occurred symbols.

- Our solution to the oversensitive detection issue is a stochastic language $\tilde{L}=\{T \mid P(T)>$ $\eta$ \}, which estimates proper boundaries of normal behaviors from training samples. In our stochastic language, each tested trace $T$ yields a probability showing how likely $T$ can be accepted by $\tilde{L}$. A threshold $\eta \in[0,1]$ is used to decide the acceptance of the trace by $\tilde{L}$.

Our program anomaly detection model is defined as a stochastic mildly context-sensitive language $\tilde{L}$ in Definition 2.3.

Definition 2.3. $\tilde{L}=\left\{T \mid\left(T \in \Sigma^{*}\right) \wedge(P(T)>\eta) \wedge\left(S \Rightarrow^{+} T\right)\right\}$, where $\Sigma=\left\{e_{0}, e_{1}, e_{2}, \ldots\right\}$ is the set of all events monitored; $S \Rightarrow^{+} T$ is the string generation operation that appends a symbol to the left or right of an existing string/trace; all strings start from an empty string $S=\{\epsilon\}$; the acceptance of a string/trace is checked by $P(T)>\eta$, which verifies event frequency relation in a string.

We give an example of $\tilde{L}$ in Table 1 . The detection system accepts program traces as normal when (i) the trace contains same numbers of $s_{1}$ and $s_{2}$, and (ii) the number of $s_{3}$ in the trace is higher than half of the number of $s_{1}$. The acceptance rule of a specific language is customized by 
the $P(T)$ function, which can characterize both the event co-occurrence relation and the event occurrence frequency relation.

\section{LAD SYSTEM OVERVIEW}

We present $\mathrm{LAD}$, a detection system to embody the stochastic mildly context-sensitive language $\tilde{L}$ discussed in Section 2.3. The goal of the detection system is to fulfill both the (binary) event cooccurrence analysis and the (quantitative) event occurrence frequency analysis supported by $\tilde{L}$.

We present an overview of LAD for analyzing long program traces in this section. We develop a constrained agglomerative clustering algorithm to overcome the behavior diversity challenge. We develop a compact and fixed-length matrix representation to profile long program traces. The matrix representation only records relational information among events. It is an implementation to avoid exponential training convergence time as discussed in $\tilde{L}$.

\subsection{Profiling Program Behaviors}

We describe the profiling procedure in LAD using the example event set: $\left\{c a l l, \operatorname{ret}^{3}\right\}^{4}$. call and ret expose user-space program activities and provide a more detailed understanding of the program execution than system calls. Function calls are exposed in some automata-based methods, e.g., Dyck model [19], but for a different purpose, i.e., eliminating non-deterministic paths.

We denote the overall activity of a program within a long trace segment $T^{5}$ as a behavior instance $b$. Instance $b$ recorded in $T$ is profiled in two matrices:

Definition 3.1. An event co-occurrence matrix $O$ is an $m \times n$ Boolean matrix recording cooccurred call events in a behavior instance $b . o_{i, j}=$ True indicates the occurrence of the call from the $i$-th row symbol (a routine) to the $j$-th column symbol (a routine). Otherwise, $o_{i, j}=$ False.

Definition 3.2. A transition frequency matrix $F$ is an $m \times n$ non-negative matrix containing occurrence frequencies of all calls in a behavior instance $b . f_{i, j}$ records the occurrence frequency of the call from the $i$-th row symbol (a routine) to the $j$-th column symbol (a routine). $f_{i, j}=0$ if the corresponding call does not occur in $O$.

$$
O_{m, n}=\left[\begin{array}{cccc}
o_{1,1} & o_{1,2} & \cdots & o_{1, n} \\
o_{2,1} & o_{2,2} & \cdots & o_{2, n} \\
\vdots & \vdots & \ddots & \vdots \\
o_{m, 1} & o_{m, 2} & \cdots & o_{m, n}
\end{array}\right] \quad F_{m, n}=\left[\begin{array}{cccc}
f_{1,1} & f_{1,2} & \cdots & f_{1, n} \\
f_{2,1} & f_{2,2} & \cdots & f_{2, n} \\
\vdots & \vdots & \ddots & \vdots \\
f_{m, 1} & f_{m, 2} & \cdots & f_{m, n}
\end{array}\right]
$$

For one specific $b, O$ is a Boolean interpretation of $F$ that

$$
o_{i, j}=\left\{\begin{array}{lll}
\text { True } & \text { if } & f_{i, j}>0 \\
\text { False } & \text { if } & f_{i, j}=0
\end{array}\right.
$$

$O$ and $F$ are succinct representations of the dynamic call graph of a running program. $m$ and $n$ are total numbers of possible callers and callees in the program, respectively. Row/column symbols in $O$ and $F$ are determined through static analysis. $m$ may not be equal to $n$, in particular, when calls inside libraries are not counted.

\footnotetext{
${ }^{3}$ ret is paired with call, which can be verified via existing control-flow integrity (CFI) technique. We do not involve the duplicated correlation analysis of ret in our model, but we trace ret to mark function boundaries for long trace partitioning (discussed in Section 5 and Section 6.1).

${ }^{4} \Sigma$ is larger than the set of two because call/ret with different addresses are different events/symbols in $\Sigma$.

${ }^{5}$ The definition of $T$ should be specified by security analysts or software developers. Automatic definition construction could be developed to accelerate the deployment of the system.
} 


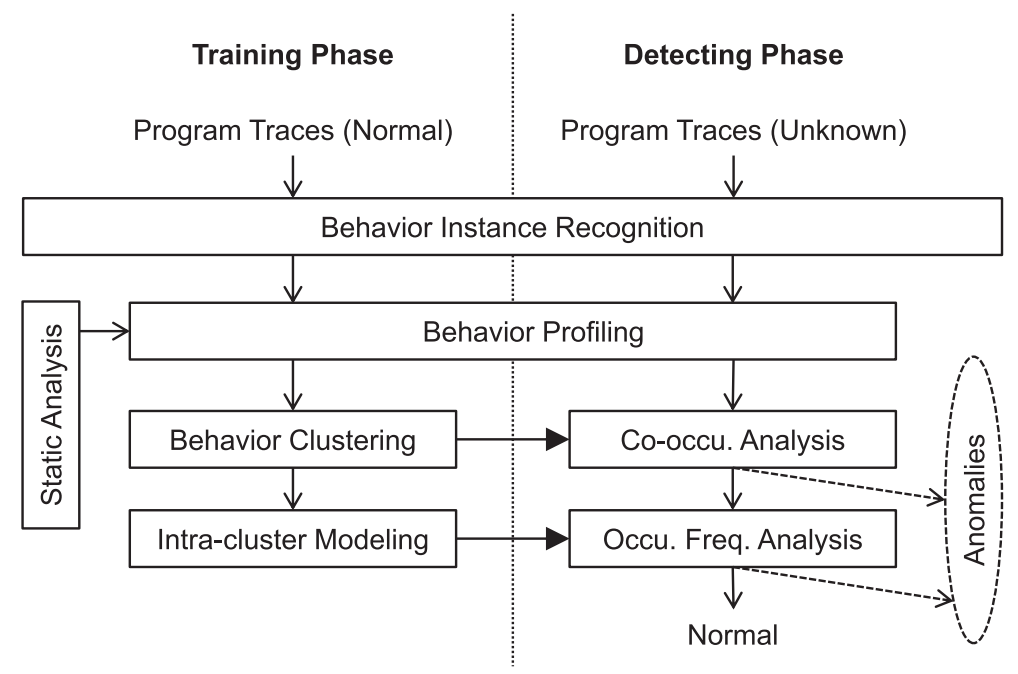

Fig. 3. Information flows among operations in two stages and two phases of LAD.

Bitwise operations, such as AND, OR, and XOR apply to co-occurrence matrices. For example, $O^{\prime}$ AND $O^{\prime \prime}$ computes a new $O$ that $o_{i, j}=o_{i, j}^{\prime}$ AND $o_{i, j}^{\prime \prime}$.

Profiles at different granularities. Although designed to be capable of modeling user-space program activities via function calls, LAD can also digest coarse level program traces for learning program behaviors. For example, system calls can be traced and profiled into $O$ and $F$ to avoid excessive tracing overheads in performance-sensitive deployments. The semantics of the matrices change in this case; each cell in $O$ and $F$ represents a statistical relation between two system calls. The detection is not as accurate as our standard design because system calls are coarse descriptions of program executions.

\subsection{Architecture}

LAD consists of two complementary stages of modeling and detection for event co-occurrence analysis and event occurrence frequency analysis, respectively.

The first stage models the binary representation of event co-occurrences in a long program trace via event co-occurrence matrix $O$ for event co-occurrence analysis. It consists of a training operation Behavior Clustering and a detection operation Co-occurrence Analysis.

The second stage models the quantitative frequency relation among events in a long trace via transition frequency matrix $F$ for event occurrence frequency analysis. It consists of a training operation Intra-Cluster Modeling and a detection operation Occurrence Frequency AnAlysis.

We illustrate the architecture of LAD in Figure 3 and brief each operation below.

(1) Behavior Profiling recognizes target long trace segments $\left\{T_{1}, T_{2}, \ldots\right\}$ in raw traces and profiles $b$ from each $T$ into $O$ and $F$. Symbols in $F$ and $O$ are retrieved via static program analysis or system call table lookup.

(2) Behavior Clustering is a training operation. It takes in all normal behavior instances $\left\{b_{1}, b_{2}, \ldots\right\}$ and outputs a set of behavior clusters $\mathbb{C}=\left\{C_{1}, C_{2}, \ldots\right\}$ where $C_{i}=$ $\left\{b_{i_{1}}, b_{i_{2}}, \ldots\right\}$ and $b_{i} \in\left\{b_{1}, b_{2}, \ldots\right\}$. 
(3) Intra-Cluster Modeling is a training operation. It is performed in each cluster. It takes in all normal behavior instances $\left\{b_{i_{1}}, b_{i_{2}}, \ldots\right\}$ for $C_{i}$ and constructs one deterministic model and one probabilistic model for computing the refined normal boundary in $C_{i}$.

(4) Co-occurrence Analysis is an inter-cluster detection operation that analyzes $O$ (of $b$ ) against clusters in $\mathbb{C}$. If behavior instance $b$ is normal, it reduces the detection problem to subproblems within a set of behavior clusters $\mathbb{C}_{b}=\left\{C_{b_{1}}, C_{b_{2}}, \ldots\right\}$, in which $b$ closely fits.

(5) Occurrence Frequency Analysis is an intra-cluster detection operation that analyzes $F$ (of $b$ ) in each $C_{b}$. Behavior instance $b$ is normal if $F$ abides by the rules extracted from $C_{b}$ and $F$ is within the normal boundary established in $C_{b}$.

\section{INTER-/INTRA-CLUSTER DETECTION}

We detail the two stages of the training and modeling procedures in LAD. The first stage contains a constrained clustering algorithm, which differentiates diverse program behaviors, divides the detection problem into subproblems, and performs the first round dimensionality reduction. The clustering enables inter-/intra-cluster detection in the first/second stage, respectively.

\subsection{Behavior Clustering (Training)}

We develop a constrained agglomerative clustering algorithm that addresses two special needs to handle program behavior instances for anomaly detection: (i) long tail elimination, and (ii) borderline behavior treatment. Agglomerative clustering is a bottom-up hierarchical clustering strategy that merges nearest clusters till stopping criteria, e.g., distance of clusters, number of clusters. It requires little knowledge about the results before operating, and the algorithm is flexible to adjust. Standard agglomerative clustering algorithms result in a large number of tiny clusters in a long-tail distribution (shown in Section 6.1). Tiny clusters do not provide sufficient numbers of samples for statistical learning of the refined normal boundary inside each cluster. Standard algorithms also do not handle borderline behaviors, which could be trained in one cluster and tested in another, resulting in false alarms.

Our algorithm (Algorithm 1) clusters program behavior instances based on the co-occurred events in behavior instances. Our algorithm handles the borderline behavior issue-behavior instances on the borderlines are randomly selected into clusters-with a two-step process:

(1) generate scopes of clusters in an agglomerative way (lines 11-25)

(2) add behavior instances to generated clusters (lines 27-39)

Our algorithm initializes clusters as individual behavior instances (line 2 to line 10), then the nearest clusters are merged (line 11 to line 25) till stopping criteria are reached (line 13) in the first step. The scope of each cluster is calculated in the first step, but not the items in each cluster. Step two fills each cluster with behavior instances and handles borderline behavior (lines 27-39).

We use a lazily updated heap $h$ in Algorithm 1 to minimize the calculation and sorting of distances between intermediate clusters. Each entry in $h$ contains the distance between two clusters, and $h$ is sorted based on the distance. The design of the lazily updated heap ensures that a previously merged cluster is not removed proactively in $h$ until the entry containing it is popped and abandoned. Algorithm 1 performs lazy removal of dead clusters in $h$. Dead clusters refer to the clusters that are merged into others and no longer exist.

The scope of a cluster $C=\left\{b_{i} \mid 0 \leq i \leq k\right\}$ is represented by its event co-occurrence matrix $O_{C}$. $O_{C}$ records occurred events in any behavior instances in $C$. It is defined in Equation (2) where $O_{b_{i}}$ is the event co-occurrence matrix of $b_{i}$.

$$
O_{C}=O_{b_{1}} \text { OR } O_{b_{2}} \text { OR } \ldots \text { OR } O_{b_{k}}, 0 \leq i \leq k
$$




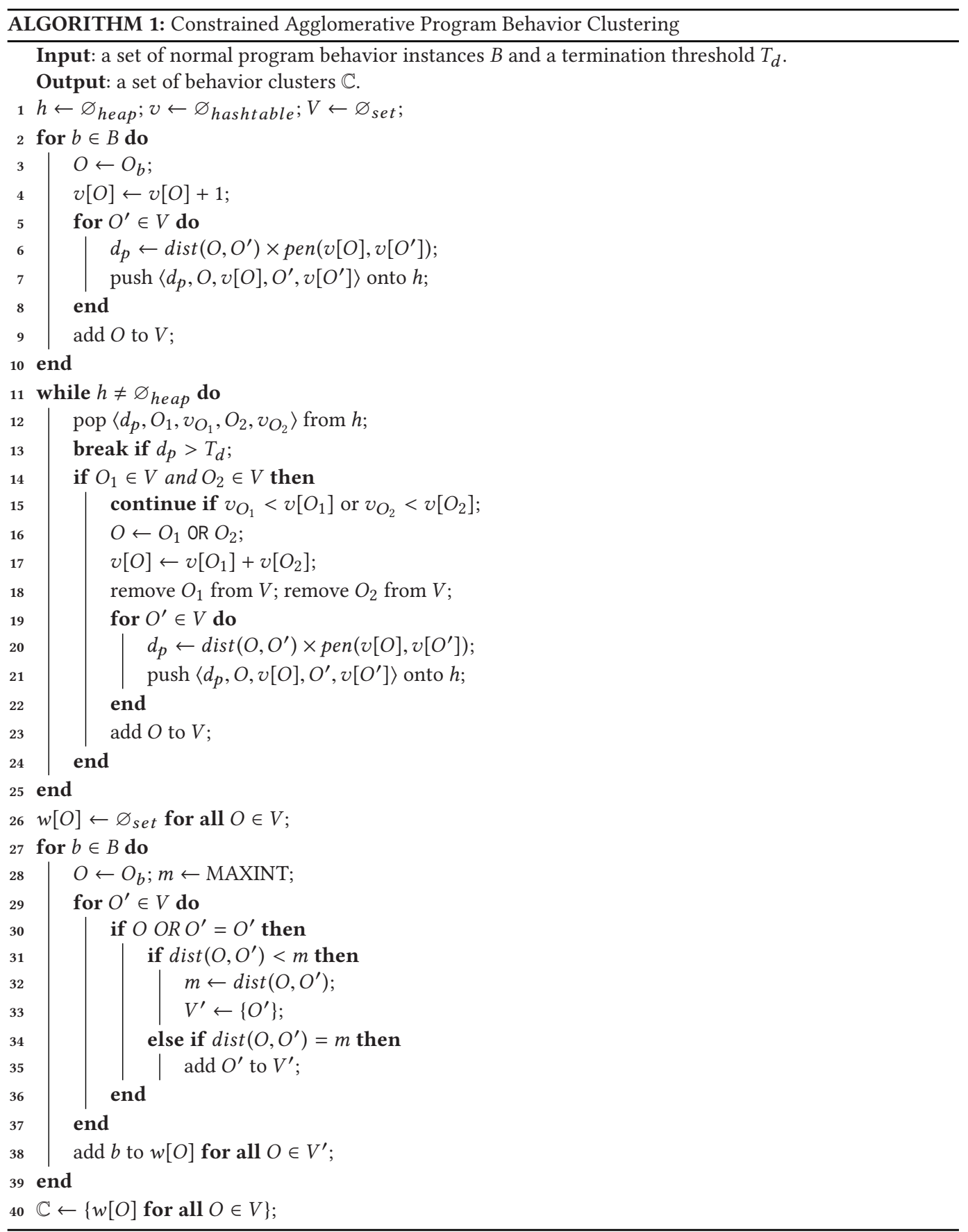

dist(): distance function between behaviors/clusters. pen(): penalty function for long tail elimination. 
The distances between (i) two behavior instances, (ii) two clusters, and (iii) a behavior instance and a cluster are all measured by their co-occurrence matrices $O_{1}$ and $O_{2}$ in Equation (3), where $|O|$ counts the number of True in $O$.

$$
\operatorname{dist}\left(O_{1}, O_{2}\right)=\frac{\operatorname{Hamming}\left(O_{1}, O_{2}\right)}{\min \left(\left|O_{1}\right|,\left|O_{2}\right|\right)}
$$

Hamming distance alone is insufficient to guide the cluster agglomeration: it loses the semantic meaning of $O$, and it weighs True and False the same. However, in co-occurrence matrices, only True contributes to the co-occurrence of events.

For example, given four $O$ s

$$
O_{1}=\left[\begin{array}{ll}
1 & 1 \\
1 & 1
\end{array}\right], O_{2}=\left[\begin{array}{ll}
0 & 1 \\
1 & 1
\end{array}\right], O_{3}=\left[\begin{array}{ll}
0 & 0 \\
1 & 1
\end{array}\right], O_{4}=\left[\begin{array}{ll}
0 & 0 \\
0 & 1
\end{array}\right],
$$

$O_{1}$ and $O_{2}$ are nearer than $O_{3}$ and $O_{4}\left(\operatorname{dist}\left(O_{1}, O_{2}\right)=33 \%<\operatorname{dist}\left(O_{3}, O_{4}\right)=100 \%\right)$, while their pure Hamming distances are equal (Hamming $\left.\left(O_{1}, O_{2}\right)=\operatorname{Hamming}\left(O_{3}, O_{4}\right)=1\right)$.

We explain the unique features of our constrained agglomerative clustering algorithm over the standard design as follows.

- Long tail elimination. A standard agglomerative clustering algorithm produces clusters with a long tail distribution of cluster sizes-there are a large number of tiny clusters, and the unbalanced distribution remains at various clustering thresholds. Tiny clusters provide insufficient number of behavior instances to train probabilistic models in INTRA-CLUSTER MODELING.

In order to eliminate tiny/small clusters in the long tail, our algorithm penalizes $\operatorname{dist}\left(O_{1}, O_{2}\right)$ by Equation (4) before pushing it onto $h .\left|C_{i}\right|$ denotes the size of cluster $C_{i}$.

$$
\operatorname{pen}\left(\left|C_{1}\right|,\left|C_{2}\right|\right)=\max \left(\log \left(\left|C_{1}\right|\right), \log \left(\left|C_{2}\right|\right)\right)
$$

- Penalty maintenance: The distance penalty between $C_{1}$ and $C_{2}$ changes when any size of $C_{1}$ and $C_{2}$ changes. In this case, all entries in $h$ containing a cluster whose size changes should be updated or nullified.

We use a version control to mark the latest and deprecated versions of clusters in $h$. The version of a cluster $C$ is recorded as its current size (an integer). It is stored in $v[O]$ where $O$ is the event co-occurrence matrix of $C$. $v$ is a hashtable that assigns 0 to an entry when the entry is accessed for the first time. A heap entry contains two clusters, their versions, and their distance when pushed to $h$ (line 7 and line 21). An entry is abandoned if any of its two clusters are found deprecated at the moment the entry is popped from $h$ (line 15).

- Borderline behavior treatment: It may generate a false positive when (i) $\operatorname{dist}\left(b, C_{1}\right)=$ $\operatorname{dist}\left(b, C_{2}\right)$, (ii) $b$ is trained only in $C_{1}$ during InTra-CLuster Modeling, and (iii) a similar behavior instance $b^{\prime}$ is tested against $C_{2}$ in operation Occurrence Frequency Analysis (intra-cluster detection).

To treat the borderline behaviors correctly, our clustering algorithm duplicates $b$ in every cluster, to which $b$ may belong (line 27-39). This operation also increases cluster sizes and results in sufficient training in INTRA-CLUSTER MODELING.

\subsection{Co-Occurrence Analysis (Detection)}

This operation performs inter-cluster detection to seek event co-occurrence anomalies. A behavior instance $b$ is tested against all normal clusters $\mathbb{C}$ to check whether the co-occurred events in $b$ are consistent with co-occurred events found in a single cluster. An alarm is raised if no such 
cluster is found. Otherwise, $b$ and its most closely fitted clusters $\mathbb{C}_{b}=\left\{C_{1}, \ldots, C_{k}\right\}$ are passed to Occurrence Frequency AnAlysis for intra-cluster detection.

An incoming behavior instance $b$ fits in a cluster $C$ if $\left(O_{b}\right.$ OR $\left.O_{C}=O_{C}\right) \wedge\left(O_{b}\right.$ AND $\left.O_{C}^{*}=O_{C}^{*}\right)$ where $O_{C}$ and $O_{b}$ are the event co-occurrence matrices of $C$ and $b$, and $O_{C}^{*}$ is a feature of $C$ defined in Equation (5). We define the fitting test to fulfill the event co-occurrence analysis and detect anomalous behaviors with fabricated or broken co-occurrences.

$$
O_{C}^{*}=O_{b_{1}} \text { AND } O_{b_{2}} \text { AND } \ldots \text { AND } O_{b_{k}}, 0 \leq i \leq k
$$

The detection process searches for all clusters in which $b$ fits, i.e., $\mathbb{C}_{b}$. If $\mathbb{C}_{b} \neq \varnothing$, distances between $b$ and each cluster in $\mathbb{C}_{b}$ are calculated using Equation (3). The clusters with the nearest distance $\left(\left|\mathbb{C}_{b}\right| \geq 1\right)$ are selected as $\mathbb{C}_{b}$.

\subsection{Intra-Cluster Modeling (Training)}

Within a cluster $C$, LAD analyzes behavior instances through their transition frequency matrices $\left\{F_{b} \mid b \in C\right\}$. The matrices are vectorized into data points in a high-dimensional detection space where each dimension records the occurrence frequency of a specific event across profiles. Two analysis methods reveal relations among frequencies.

The probabilistic method. We employ a one-class SVM, i.e., $v$-SVM [48], to seek a frontier $\mathcal{F}$ that envelops all behavior instances $\{b \mid b \in C\}$.

(a) Each frequency value is preprocessed with a logarithmic function $f(x)=\log _{2}(x+1)$ to reduce the variance between extreme values (empirically proved necessary).

(b) A subset of dimensions are selected through frequency variance analysis (FVA) ${ }^{6}$ or principle component analysis (PCA) ${ }^{7}$ [43] before data points are consumed by $v$-SVM. This step manages the curse of dimensionality, a common concern in high-dimensional statistical learning.

(c) We pair the $v$-SVM with a kernel function, i.e., radial basis function (RBF), ${ }^{8}$ to search for a non-linearly $\mathcal{F}$ that envelops $\{b \mid b \in C\}$ tightly. The kernel function transforms a non-linear separating problem into a linearly separable problem in a high-dimensional space.

The deterministic method. We employ variable range analysis to measure frequencies of events with zero or near zero variances across all program behaviors $\{b \mid b \in C\}$.

Frequencies are discrete integers. If all frequencies of an event in different behavior instances are the same, PCA simply drops the corresponding dimension. In some clusters, all behavior instances (across all dimensions) in $C$ are the same or almost the same. Duplicated data points are treated as a single point, and they cannot provide sufficient information to train probabilistic models, e.g., one-class SVM.

Therefore, we extract deterministic rules for events with zero or near zero variances. This model identifies the frequency range $\left[f_{\min }, f_{\max }\right]$ for each of such events. $f_{\min }$ can equal to $f_{\max }$.

\subsection{Occurrence Frequency Analysis (Detection)}

This operation performs intra-cluster detection to seek quantitative frequency relational anomalies: (i) deviant relations among multiple event occurrence frequencies, and/or (ii) aberrant

\footnotetext{
${ }^{6}$ FVA selects dimensions/events with larger-than-threshold frequency variances across all behavior instances in $C$.

${ }^{7} \mathrm{PCA}$ selects linear combinations of dimensions/events with larger-than-threshold frequency variances, which is a generalization of FVA.

${ }^{8}$ Multiple functions have been tested for selection.
} 
occurrence frequencies. Given a program behavior instance $b$ and its closely fitted clusters $\mathbb{C}_{b}=$ $\left\{C_{1}, \ldots, C_{k}\right\}$ discovered in Co-occurrence AnALYsis, this operation tests $b$ in every $C_{i}(0 \leq i \leq k)$ and aggregates the results using Equation (6).

$$
\exists C \in \mathbb{C} N_{c l t}(b, C) \Rightarrow b \text { is normal }
$$

The detection inside $C$ is performed with three rules, and the result is aggregated into $N_{c l t}(b, C)$ through logical conjunction of available rules: ${ }^{9}$

$$
N_{c l t}(b, C) \leftarrow \text { True iff } b \text { is tested normal by all applicable rules to } C \text {. }
$$

-Rule 1: normal if the behavior instance $b$ passes the probabilistic model detection. The frequency transition matrix $F$ of $b$ is vectorized into a high-dimensional data point and tested against the one-class SVM model built in INTRA-CLUSTER ModeLING. This operation computes the distance $d$ between $b$ and the frontier $\mathcal{F}$ established in the $v$-SVM. If $b$ is within the frontier or $b$ is on the same side as normal behavior instances, then $d>0$. Otherwise, $d<0 . d$ is compared with a detection threshold $T_{f}$ that $T_{f} \in(-\infty,+\infty) . b$ is abnormal if $d<T_{f}$.

- Rule 2: normal if the behavior instance $b$ passes the deterministic model detection. Events in $b$ with zero or near zero variances are tested against the deterministic model built in INTRACLUSTER Modeling. $b$ is abnormal if any event frequency of $b$ exceeds its normal range.

- Rule 3: presumption of innocence in tiny clusters. If no frequency model is trained in $C$ because the size of $C$ is too small, the behavior instance $b$ is marked as normal. This rule sacrifices the detection rate for reducing false alarms in insufficiently trained clusters.

\section{IMPLEMENTATION}

We implement a prototype of LAD on Linux (Fedora 21, kernel 3.19.3). The static analysis is realized through C (ParseAPI [42]). The profiling, training, and detection phases are realized in Python. The dynamic tracing and behavior recognition are realized through Intel Pin, a leading dynamic binary instrumentation framework, and SystemTap, a low-overhead dynamic instrumentation framework for Linux kernel. Tracing mechanisms are independent of our detection design; more efficient tracing techniques can be plugged in, replacing Pin and SystemTap to improve the overall performance in the future.

Static analysis before profiling: symbols and address ranges of routines/functions are discovered for programs and libraries. The information helps to identify routine symbols if not found explicitly in dynamic tracing. Moreover, we leverage static analysis to list legal caller-callee pairs.

Profiling: Our prototype (i) verifies the legality of events (function calls) in a behavior instance $b$ and (ii) profiles $b$ into two matrices (Section 3.1). The event verification filters out simple attacks that violate control flows before our approach detects stealthy aberrant path attacks. We implement profile matrices in Dictionary of Keys (DOK) format to minimize storage space for sparse matrices.

Dynamic tracing and behavior recognition: We develop a Pintool in JIT mode to trace function calls in the user space and to recognize boundaries of long trace segments within entire program executions. Our Pintool is capable of tracing (i) native function calls, (ii) library calls (iii) function calls inside dynamic libraries, (iv) kernel thread creation and termination. Traces of different threads are isolated and stored separately. Our Pintool recognizes whether a call is made within a given routine and on which nested layer the given routine executes (if nested execution of the

\footnotetext{
${ }^{9}$ Not all three rules could be applicable to an arbitrary cluster $C$.
} 
Table 2. The Profile Information of Programs/Libraries and Statistics of Normal Profiles

\begin{tabular}{|c|c|c|c|c|c|c|c|}
\hline \multirow[b]{2}{*}{ Program } & \multirow[b]{2}{*}{ Version } & \multicolumn{3}{|c|}{ Profile Overview } & \multicolumn{3}{|c|}{ Average Normal Profile } \\
\hline & & Event Set & Segmentation & \#(N.P.) & $\#($ Sym.) & \#(Event) & \#(U.E.) \\
\hline sshd & 1.2 .30 & function calls & routine boundary & 4,800 & 415 & 34,511 & 180 \\
\hline libpcre & 8.32 & function calls & library call & 17,360 & 79 & 44,893 & 45 \\
\hline sendmail & 8.14 .7 & system calls ${ }^{\dagger}$ & event statistics & 6,579 & 350 & 1,134 & 213 \\
\hline
\end{tabular}

N.P., Sym., and U.E. are short for normal profile, symbol, and unique event, respectively.

An event is a function or system call (detailed in column Event Set).

A normal profile is recorded in a matrix $O$ and a matrix $F$.

A symbol defines a caller or a callee of an event.

† Function calls are not traced due to their complex process spawning logic. It requires customization of our Pintool to trace them.

given routine occurs). This functionality enables the recognition of long trace segments through routine boundary partitioning.

We demonstrate that our approach is versatile, recognizing program behaviors at different granularities. We develop a SystemTap script to trace system calls with timestamps. It enables long trace partitioning via activity intervals when the program is monitored as a black box.

\section{EVALUATIONS}

We extensively evaluate the detection accuracy, capability, and performance of our LAD system. We aim to answer the following questions in this section:

(1) How well does our approach detect real-world aberrant path attacks? (Section 6.2)

(2) How accurate is our approach at recognizing anomalous program behavior instances? (Section 6.3)

(3) What's the advantage of our constrained agglomerative clustering algorithm? (Section 6.4)

(4) How much overhead does our detection incur? (Section 6.5)

\subsection{Experiment Setup}

We study three programs/libraries (Table 2) in distinct categories. We demonstrate that LAD is a versatile detection solution to be applied to programs and dynamic libraries with various event definitions and long trace segmentation means. We detail the programs/libraries and their training dataset (normal profiles) below.

[sshd] Long trace segment definition: all function calls within routine do_authentication() of sshd (SLOC $=19,215) .{ }^{10}$ The routine do_authentication() is called in a forked thread after a client initializes its connection to sshd. All session activities occur within the long trace segment. Normal runs cover three authentication methods (password, public key, rhosts), each of which contains 800 successful and 800 failed connections. 128 random commands are executed in each successful connection. [libpcre] Long trace segment definition: all function calls inside libpcre when a library call is made and control flows go into libpcre $(\mathrm{SLOC}=68,017)$. Library calls are triggered through grep -P. Over 10,000 normal tests are used from the libpcre package.

\footnotetext{
${ }^{10}$ The actually monitored part accessible from do_authentication( $)$ is smaller than the entire program.
} 
Table 3. Overview of Program Behavior Clustering

\begin{tabular}{|c|c|c|c|}
\hline$\overline{T_{d}}$ & 1.6 & 2.0 & 1.6 \\
\hline$|\mathbb{C}|$ & 6 & 29 & 20 \\
\hline
\end{tabular}

Table 4. Overview of Reproduced Attacks and Detection Results

\begin{tabular}{lllrrr}
\hline Attack & Target & \multicolumn{1}{c}{ Attack Settings } & an(A.) & D.R. & FPR $^{\mathrm{u}}$ \\
\hline $\mathrm{FVO}^{a}$ & sshd & an inline virtual exploit that matches a username & 800 & $100 \%$ & 0.0001 \\
$\mathrm{ReDoS}$ & libpcre & 3 deleterious patterns paired with 8-23 input strings & 46 & $100 \%$ & 0.0001 \\
$\mathrm{DHA}^{b}$ & sendmail & probing batch sizes: 8, 16, 32, 64, 100, 200, and 400 & 14 & $100 \%$ & 0.0001 \\
\hline
\end{tabular}

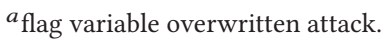

$b$ directory harvest attack.

Note: A. is short for attack attempt. D.R. is short for detection rate. $\mathrm{FPR}^{u}$ is the false positive rate upper bound (details in Section 6.1).

[sendmail] Long trace segment definition: a continuous system call sequence wrapped by long no-op (no system call) intervals. sendmail is an event-driven program that only emits system calls when sending/receiving emails or performing a periodical check. We set up this configuration to demonstrate that LAD can consume various events, e.g., system calls. We collect over 6,000 normal profiles on a public sendmail server for 8 hours.

We list clustering threshold $T_{d}$ used for the three studied programs/libraries in Figure $3 .{ }^{11}|\mathbb{C}|$ denotes the number of clusters computed with the specific $T_{d}$.

In operation Occurrence Frequency Analysis, the detection threshold $T_{f}$ is determined by a given false-positive rate (FPR) upper bound, i.e., $\mathrm{FPR}^{u}$, through cross-validation. In the training phase of cross-validation, we perform multiple random 10-fold partitioning. Among distances from all training partitions, $T_{f}$ is initialized as the $k$ th smallest distance within distances ${ }^{12}$ between a behavior instance and the $v$-SVM frontier $\mathcal{F} . k$ is calculated using $\mathrm{FPR}^{u}$ and the overall number of training cases. The FPR is calculated in the detection phase of cross-validation. If FPR $>\mathrm{FPR}^{u}$, a smaller $k$ is selected until FPR $\leq \mathrm{FPR}^{u}$.

\subsection{Detecting Real-World Attacks}

We reproduce three known aberrant path attacks to test the detection capability of our LAD system. Our prototype detects all attack attempts, and the detection overview is in Table 4.

Flag Variable Overwritten Attack (FVO in Table 4) is a non-control data attack. An attacker tampers with decision-making variables. The exploit takes effect when the manipulated data affects the control flow at some later point of execution.

We reproduce the flag variable overwritten attack against sshd introduced by Chen et al. [6]. We describe the attack in Section 2.1, line (a) and in Figure 1. We simplify the attack procedure by placing an inline virtual exploit in sshd right after the vulnerable routine packet_read():

$$
\text { if (user }[0]==\text { 'e' \&\& user[1] == 'v' \&\& user[2] == 'e') authenticated = 1; }
$$

\footnotetext{
${ }^{11}$ The value is empirically chosen to keep a balance between an effective recognition of diverse behaviors and an adequate elimination of tiny clusters.

${ }^{12}$ The distance can be positive or negative. More details are specified in Rule 1 (Section 4.4).
} 
Table 5. Samples of Normal and Anomalous sshd Traces

\begin{tabular}{lll}
\hline Normal & \multicolumn{1}{c}{ Normal } & \multicolumn{1}{c}{ Attack } \\
\hline (successfully authenticated) & (failed authentication) & (flag variable overwritten attack) \\
$\ldots$ & $\ldots$ & $\ldots$ \\
auth_p $>$ xfree & auth_p $>$ xfree & auth_p $>$ xfree \\
do_auth $>$ xfree & do_auth $>$ debug & do_auth $>$ debug \\
do_auth $>$ log_msg & do_auth $>$ xfree & do_auth $>$ xfree \\
do_auth $>$ p_start & do_auth $>$ p_start & do_auth $>$ p_start \\
p_start $>$ buf_clr & p_start $>$ buf_clr & p_start $>$ buf_clr \\
$\ldots$ & $\ldots$ & $\ldots$ \\
phdtw $>$ buf_len & phdtw $>$ buf_len & phdtw $>$ buf_len \\
do_auth $>$ do_autd & do_auth $>$ p_read & do_auth $>$ do_autd \\
$\ldots$ & $\ldots$ & ... \\
\hline
\end{tabular}

Note: "caller > callee" denotes a function call. Routine names are abbreviated to save space.

This inline virtual exploit produces the immediate consequence of a real exploit-overwriting authenticated. It does not interfere with our tracing/detection because no call instruction is employed. Each attack attempt is constructed with the user name "eve" and 128 random shell commands after a successful login.

Our approach (configured at $\mathrm{FPR}^{u}$ 0.0001) successfully detects all attack attempts in inter-cluster detection (Co-OCCURREnce AnAlysis). ${ }^{13}$ We present normal and attack traces inside long trace segments (selected routine do_authentication()) in Figure 5 to illustrate the detection.

In Figure 5, the Attack and Normal ${ }^{b}$ bear the same trace prior to the last line, and the Attack and Normal ${ }^{a}$ bear the same trace after (including) the last line. Our approach detects the attack with event co-occurrence analysis: the control-flow segment containing do_auth $>$ debug should not co-occur with the control-flow segment containing do_auth $>$ do_authed (and following calls) in one long trace segment.

In the traces, there are identical $218 \mathrm{call}$ events including library routines $(36 \mathrm{calls}$ excluding library ones) between the third line and the last line in Figure 5. We test an $n$-gram detection tool, and it requires at least $n=37$ to detect the specific attack without libraries routine traced. The 37gram model results in an FPR of $6.47 \%$ (the FPR of our approach is less than $0.01 \%$ ). This indicates that $n$-gram models with a large $n$ is difficult to converge at training. We do not test automatonbased detection that defines illegal transitions as anomalous behaviors. Automaton-based methods cannot detect the attack in theory since there are no illegal function calls.

Flag Variable Overwritten Attack (ReDoS in Table 4) is a service abuse attack. It exploits the exponential time complexity of a regex engine when performing backtracking. The attacks construct extreme matching cases where backtracking is involved. All executed control flows are legal. The regex engine hangs due to the extreme complexity.

We produce $46 \mathrm{ReDoS}$ attack attempts targeting libpcre ${ }^{14}$. Three deleterious patterns are used (Table 6). For each deleterious pattern, attacks are constructed with an increasing length of $a$ in the input string starting at 6, e.g., aaaaaaaab. We stop attacking libpcre at different input string lengths so that the longest hanging time periods for different deleterious patterns are about the same (a few seconds). A longer input string incurs a longer hanging time; it results in a more severe ReDoS attack than a shorter one.

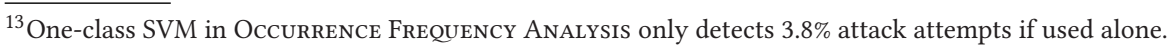

${ }^{14}$ Internal deep recursion prevention of libcpre is disabled.
} 
Table 6. Deleterious Patterns used in ReDoS Attacks

\begin{tabular}{llc}
\hline & \multicolumn{1}{c}{ Deleterious Pattern } & $\#($ Attack Attempts) \\
\hline Pattern 1 & $\wedge(a+)+\$$ & 15 \\
Pattern 2 & $((a+))+\$$ & 8 \\
Pattern 3 & $\wedge(([a-z])+)+.[A-Z]([a-z])+\$$ & 23 \\
\hline
\end{tabular}

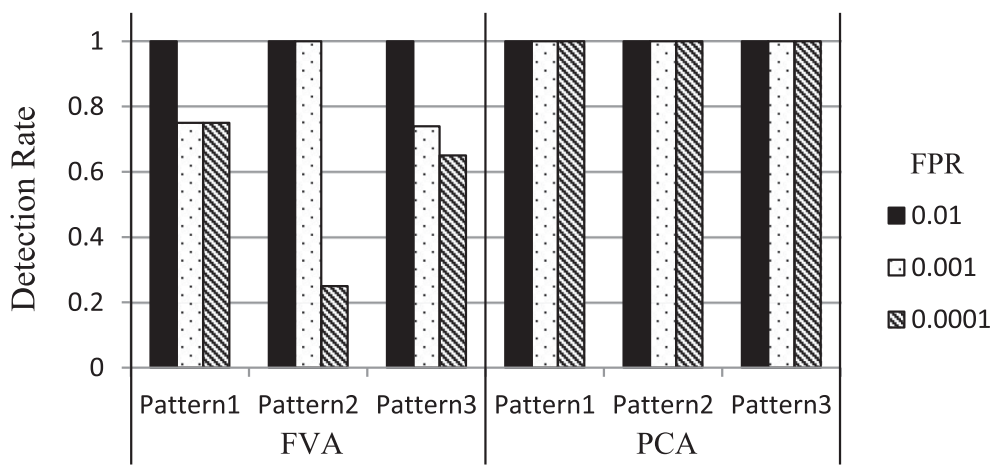

Fig. 4. Detection rates of ReDoS attacks.

ReDoS attacks are detected in intra-cluster detection operation (OcCURRENCE Frequency ANALYSIS) by the probabilistic method, i.e., $v$-SVM. We test our LAD system with both PCA and FVA feature selection (Section 4.3, the probabilistic method, line b). The detection results (Figure 4) show that LAD configured with PCA is more sensitive than it configured with FVA. LAD (with PCA) detects all attack attempts at different FPRs ${ }^{15}$. The undetected attack attempts (with FVA) are all constructed with the small amount of a in the input strings, which do not result in very severe ReDoS attacks.

Directory Harvest Attack (DHA in Table 4) is a service abuse attack. It probes valid email users through brute force. We produce $14 \mathrm{DHA}$ attack attempts targeting sendmail. Each attack attempt consists of a batch of closely sent probing emails with a dictionary of possible receivers. We conduct DHA attacks with 7 probing batch sizes from 8 to 400 (Table 4). Two attack attempts are conducted for each batch size.

Our approach (configured at $\mathrm{FPR}^{u}$ 0.0001) successfully detects all attack attempts with either PCA or FVA feature selection. ${ }^{15}$ DHA attacks are detected in intra-cluster detection (OcCURRENCE Freduency ANALYsis) by the probabilistic method, i.e., $v$-SVM. The attacks bypass the inter-cluster detection (Co-OCCURRENCE ANALYSIS) because invalid usernames occur in normal training dataset.

This experiment demonstrates that our LAD system can consume coarse program behavior descriptions (e.g., system calls) to detect attacks. Most of the probing emails do not have valid receivers. They result in a different processing procedure than that for normal emails; the batch of DHA emails processed in a long trace segment gives anomalous ratios between frequencies of valid email processing control flows and frequencies of invalid email processing control flows. In sendmail, these different control flows contain different sets of system calls, so they are revealed by system call profiles. More precise detection requires the exposure of internal program activities, such as call, jump, and return instructions in the program user space.

\footnotetext{
${ }^{15} \mathrm{No}$ attack is detected if only Co-OCCURRENCE ANALYsis is performed.
} 
Table 7. Semantics of True/False Positives/Negatives

\begin{tabular}{lcc}
\hline & $\begin{array}{c}\text { Abnormal } \\
\text { Behavior }\end{array}$ & $\begin{array}{c}\text { Normal } \\
\text { Behavior }\end{array}$ \\
\hline Anomaly detected & true positive & false positive \\
No anomaly detected & false negative & true negative \\
\hline
\end{tabular}

\subsection{Systematic Accuracy Evaluation}

We systematically demonstrate how sensitive and accurate our approach is through receiver operating characteristic (ROC). Besides normal program behaviors ground truth (Section 6.1), we generate four types of synthetic aberrant path anomalies. We first construct $F^{\prime}$ for each synthetic anomalous behavior instance $b^{\prime}$, and then we use Equation (1) to derive $O^{\prime}$ (of $b^{\prime}$ ) from $F^{\prime}$.

(1) Montage anomaly: two behavior instance $b_{1}$ and $b_{2}$ are randomly selected from two different behavior clusters. For a cell $f_{i, j}^{\prime}$ in $F^{\prime}$, if one of $f_{1, j}$ (of $\left.F_{1}\right)$ and $f_{2_{i, j}}\left(\right.$ of $F_{2}$ ) is 0 , the value of the other is copied into $f_{i, j}^{\prime}$. Otherwise, one of them is randomly selected and copied.

(2) Incomplete path anomaly: random one-eighth of non-zero cells of a normal $F$ are dropped to 0 (indicating events that have not occurred) to construct $F^{\prime}$.

(3) High-frequency anomaly: three cells in a normal $F$ are randomly selected, and their values are magnified 100 times to construct $F^{\prime}$.

(4) Low-frequency anomaly: similar to high-frequency anomalies, but the values of the three cells are reduced to 1 .

To demonstrate the effectiveness of our design in handling diverse program behaviors, we compare our LAD system with a basic one-class SVM (the same $v$-SVM and same configurations, e.g., kernel function, feature selection, and parameters, as used in the INTRA-CLUSTER MODELING operation of LAD).

We present the detection accuracy results on libpcre in Figure 5, which has the most complicated behavior patterns among the three studied programs/libraries. ${ }^{16}$ In any subfigure of Figure 5, each dot is associated with a false-positive rate (multi-round 10-fold cross-validation with 10,000 test cases) and a detection rate (1,000 synthetic anomalies). We define positives and negatives as in Table 7-an anomaly is a positive.

Figure 5 shows the effectiveness of our clustering design. The detection rate of our prototype (with $\mathrm{PCA}^{17}$ ) is usually higher than 0.9 with FPR less than 0.01 . Because of diverse patterns, basic one-class SVM fails to learn tight boundaries that wrap diverse normal patterns as expected. A loose boundary results in false negatives and low detection rates.

\subsection{Constrained Agglomerative Clustering Algorithm}

We illustrate the effectiveness of our constrained agglomerative clustering algorithm design to eliminate long tails of cluster size distributions. The optimization supports adequate training in each cluster and minimizes the use of the innocence rule in Co-occurrence ANALYsis (Section 4.4). The rule may result in false negatives.

Figure 6 compares the clustering results between standard agglomerative clustering algorithm and our constrained design regarding cluster size distribution: the dotted line denotes the results

\footnotetext{
${ }^{16}$ Results of the other two programs share similar characteristics as libpcre and are not presented.

${ }^{17} \mathrm{PCA}$ proves itself more accurate than FVA in Figure 5.
} 


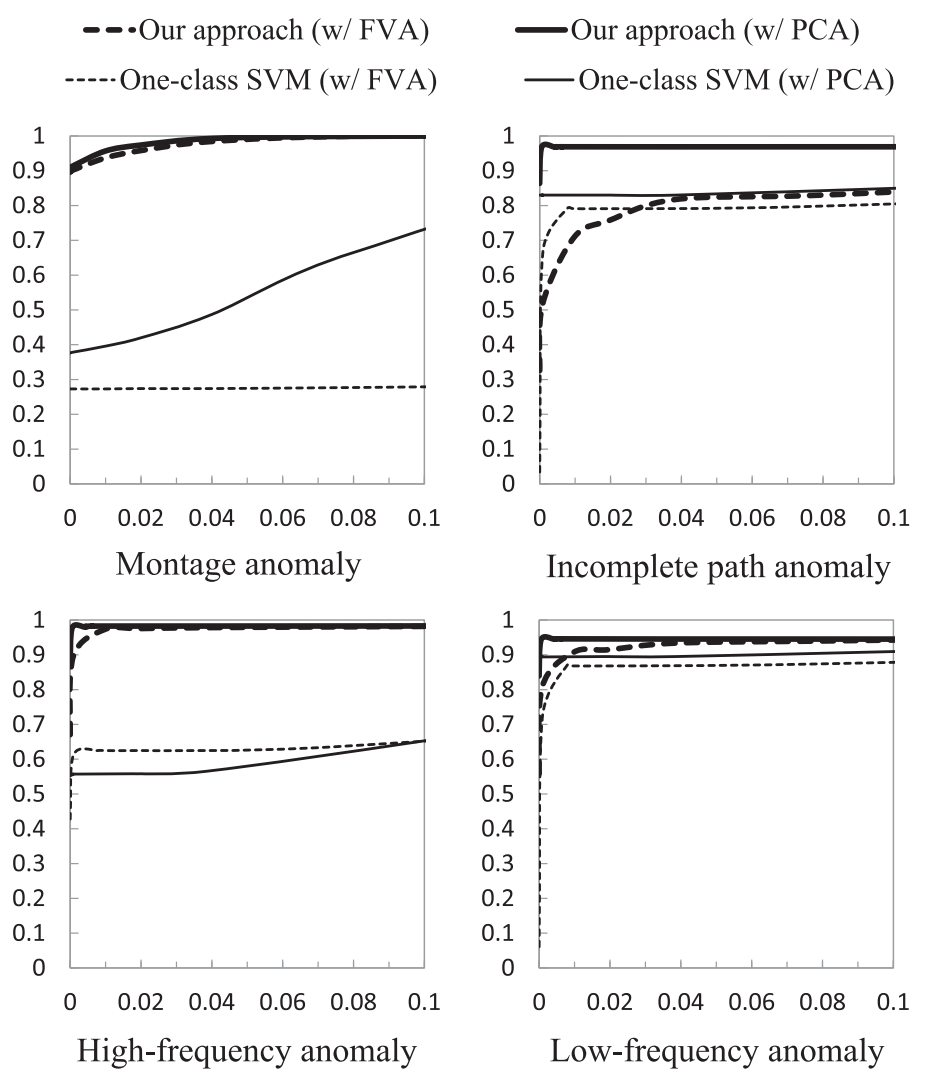

Fig. 5. libpcre ROC of LAD (our approach) vs. basic one-class SVM. X-axis is false-positive rate, and y-axis is detection rate.

of the standard agglomerative clustering algorithm as the baseline. The solid line denotes the results of the proposed constrained algorithm with size penalty. Our design eliminates tiny clusters in the long tail of cluster size distributions. Tiny clusters contain insufficient numbers of behavior instances for building probabilistic models in INTRA-CLUSTER Modeling. The constrained algorithm prioritizes the merge of tiny clusters into bigger ones by giving a cluster size penalty when calculating the distances between clusters (line 20 in Algorithm 1).

\subsection{Performance Analysis}

A fast runtime detection is important for enabling real-time protection and minimizing negative user experience [39]. The overall overhead of a program anomaly detection system comes from tracing, training, and analysis in general.

We evaluate the performance of LAD analysis procedures (inter- and intra-cluster detections) with either function call profiles (libpcre) or system call profiles (sendmail). We test the analysis on all normal profiles (libpcre: 17360, sendmail: 6579) to collect overhead for inter-cluster detection alone and the combination of inter- and intra-cluster detection. ${ }^{18}$ The analysis of each behavior instance is repeated 1,000 times to obtain a fair timing. The performance results in Figure 7 illustrate that

\footnotetext{
${ }^{18} \mathrm{PCA}$ is used for feature selection. FVA (results omitted) yields a lower overhead due to its simplicity.
} 


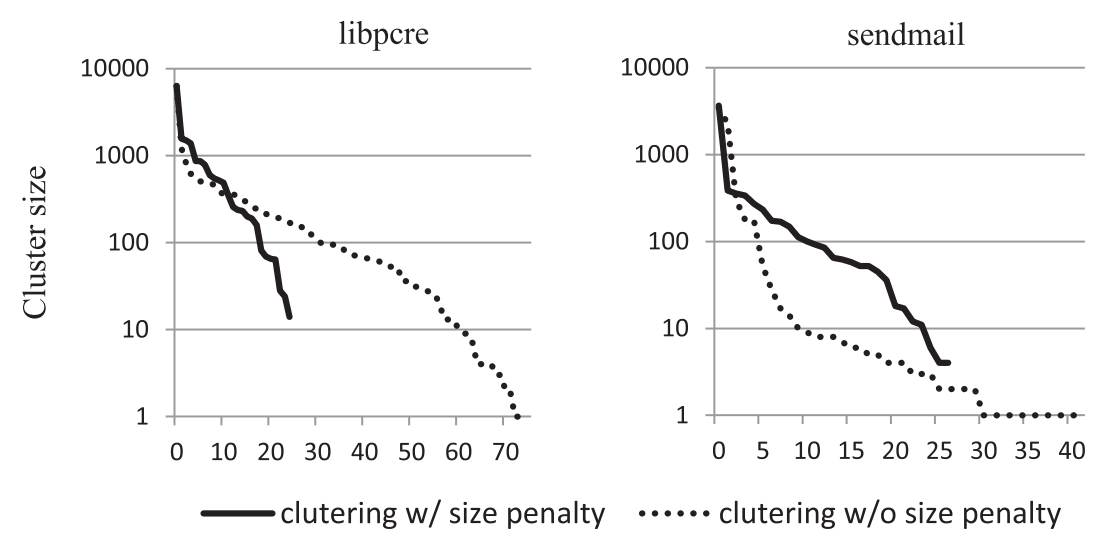

Fig. 6. Long-tail elimination with our constrained agglomerative clustering algorithm.

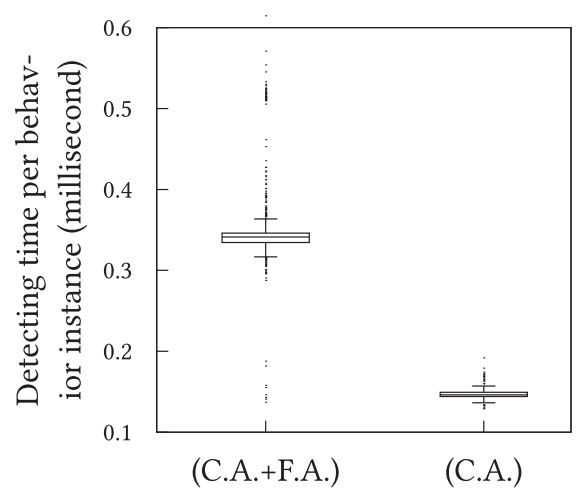

(a) libpcre

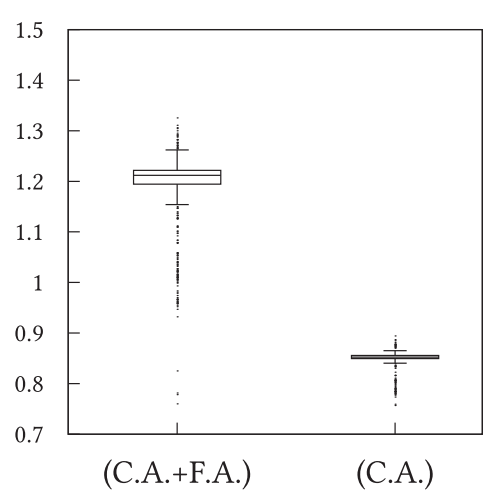

(b) sendmail

Fig. 7. Detection (analysis) overhead of our LAD system. Note: C.A.+F.A.: Inter- and intra-cluster detection combined. C.A.: Inter-cluster detection only.

-It takes $0.1 \mathrm{~ms}$ to $1.3 \mathrm{~ms}$ to analyze a single behavior instance, which contains 44,893 function calls (libpcre) or 1,134 system calls (sendmail) on average (Table 2).

- The analysis overhead is positively correlated with the number of unique events in a profile (Table 2), which is due to our DOK implementation of profile matrices.

- Anomalies detected at Co-occurrence AnAlysis takes less time to detect than anomalies detected at Occurrence Frequency Analysis because only the first stage is involved in the detection.

Two major factors affect the detection time on a program behavior instance: (i) the symbol set size, which determines the size of a profile matrix; and (ii) the number of unique events in a profile, which determines the number of non-zero entries in a matrix. Our prototype is implemented using dictionary of keys (DOK), thus the detection performance of our prototype should be affected more by the number of unique events for different programs (the last column in Table 2).

Compared with the analysis procedure, dynamic function call tracing incurs a noticeable overhead. sshd experiences a $167 \%$ overhead on average when our Pintool is loaded. A similar $141 \%$ overhead is reported by Jalan and Kejariwal in their dynamic call graph Pintool Trin-Trin [31]. 
Advanced tracing techniques, e.g., Intel Processor Tracing (PT) [33], combined with the latest hardware, e.g., Skylake architecture, can potentially reduce the tracing overhead to less than $5 \%$ toward a real-time detection system [16].

Another choice to deploy LAD is to profile program behaviors through system calls as we demonstrate using sendmail. System calls can be traced through SystemTap with near-zero overhead [57], but it sacrifices the capability to reveal user-space program activities and downgrades the modeling/detection accuracy.

LAD supports offline detection or forensics of program attacks, in which case accuracy is the main concern instead of performance [56]. Our Pintool enables analysts to locate anomalies within long program traces, and our matrices provide caller information for individual function calls. This information helps analysts quickly reduce false alarms and locate vulnerable code segments. For potential online detection deployment, tracing is the bottleneck as shown above. Finding a faster way to monitor the system, e.g., Intel PT, gives more boost to the entire procedure than detection procedure improvements. A comprehensive benchmark is also needed to evaluate the overall overhead of an online detection system in terms of different tracing mechanisms, different styles of programs, and different numbers of hooks for different monitoring needs.

Although training performance is not a typical concern for program anomaly detection, the procedure should be practical to enable the use of the approach. Our prototype shows reasonable training time from 1 to 100 seconds in all three cases we tested. The more complicated and diverse the behaviors are, the longer the algorithm takes to figure out the clusters. System calls in the sendmail case characterize full-program behaviors in 8 hours, which constructs the most diverse dataset. Over 6,000 behavior instances take $86.17 \mathrm{~s}$ to train on average. Behaviors in libpcre and sshd are defined in a more focused manner, e.g., activities within a routine or library call. They take $5.01 \mathrm{~s}$ and $1.77 \mathrm{~s}$ to train 17,360 and 4,800 behavior instances on average.

Summary. We evaluate the detection capability, accuracy, and performance of our detection prototype on Linux.

- Our approach successfully detects all reproduced aberrant path attack attempts against sshd, libpcre and sendmail with less than 0.0001 false-positive rates.

- Our approach is accurate in detecting different types of synthetic aberrant path anomalies with a high detection rate $(>0.9)$ and a low false positive rate $(<0.01)$.

- Our approach analyzes program behaviors fast; it only incurs $0.1 \mathrm{~ms}$ to $1.3 \mathrm{~ms}$ analysis overhead (excluding tracing) per behavior instance ( $1 \mathrm{k}$ to $50 \mathrm{k}$ function/system calls in our experiments).

\section{DISCUSSION}

This article aims to present a practical program anomaly detection approach at the contextsensitive language level. It does not accomplish all context-sensitive features, e.g., order of events, yet it correlates events far away and sheds light on the development towards more advanced context-sensitive detection models.

Prior to and after the presented approach, several steps can be made to complete the detection, ease the adoption, and reduce potential false positives. We first discuss potential improvements from the deployment perspective below, and then we discuss the accuracy issues as well as potential improvements.

- Fine-grained tracing with low overhead. As shown in Section 6.5, the detection of a single behavior instance (>10k events) only takes $0.1 \mathrm{~ms}$ to $1.3 \mathrm{~ms}$. What impedes the approach to be used as an online system is the tracing overhead. Practical fine-grained user-space 
tracing is the bottleneck of many program anomaly detection systems based on dynamic analysis. Fast and practical tracing mechanisms, e.g., Intel PT (discussed in Section 6.5), could open the door to online detection leveraging the proposed approach.

-Long trace segment definition. The proposed approach recognizes long trace segments based on the definitions specified by security analysts or software developers. In our evaluation, we use three simple long trace segment definitions: (i) subroutine boundary for sshd, (ii) library call boundary for libpcre, and (iii) statistical event density for sendmail. The third is the default choice if no specific knowledge is revealed about the program or attacks, yet it is the coarsest.

This ad-hoc procedure can be improved by developing a segment definition construction algorithm. The algorithm either takes in vanilla programs plus external knowledge or compiled programs with security tags specified by developers. It could list and rank generated long segment definitions to aid the deployment and configuration of the proposed approach.

-False alarms with incomplete training. We recommend deploying the proposed system for monitoring server programs or applications with relatively static behaviors. Complicated user applications such as browsers have a vast behavior space, which is difficult to cover by limited training samples. False positives could be generated if normal behaviors are not present or normal boundaries do not reach far enough to cover the tested behavior instances.

Our model discards the order information between events, which projects the original behavior space onto a lower dimensional subspace for practical modeling. However, incomplete training is still an issue for programs with dynamic behavior patterns. Further reducing the space can result in the loss of context-sensitive information and leads to less precise context-free language models like DFA models.

Since the proposed approach sacrifices precision for practicality in its design, potential mimicry attacks could be constructed to exploit the gap between $\tilde{L}$ and the most precise program execution description. Additionally, the learning-detection cycle may pose potential inaccuracy to detection results. We discuss attacks that can potentially evade the detection of our model as well as possible countermeasures.

- Exploiting the granularity of our model. An attacker may construct an attack: (i) it consists of control-flow segments without call instructions, and (ii) it does not incur future anomalous control flows containing call after exploit, e.g., only substituting arguments of legitimate exec system call to invoke /bin/sh. Our current prototype does not detect such a threat. However, this is not a theoretical defeat. The prototype can be extended to monitor and correlate non-call instructions and subprocess behaviors for detecting this attack.

- Exploiting the orderless characteristic of our model. Our approach does not enforce the order of events within a program behavior instance. The property could be exploited by an attacker. Directly adding event order information on top of our model results in upgrading $\tilde{L}$ to a linear bounded automaton (LBA), which incurs exponential training convergence time discussed in Section 2.3. It remains an open question to construct LBAs, probably with heuristics, for program anomaly detection.

- Exploiting the normal boundary of our detection. We employ a stochastic language in our approach-the one-class SVM estimates a proper boundary of normal behaviors in each of the clusters-to mitigates false positives due to insufficient training samples. The estimation opens potential opportunities for attackers to escape the detection, i.e., false negatives. 
First, an attacker can sacrifice the effectiveness of attack for not being detected. For example, in a mild brute force SSH password attack, low frequency of attack attempts makes it unlikely to be discovered. The parameter $\mathrm{FPR}^{u}$ in our prototype establishes a balance between missing attacks and oversensitive detection that yields false alarms. We provide a quantitative analysis in Section 6.3 to understand the sensitivity of our prototype and this can be further improved by choosing other boundary computation methods and parameters.

Second, if an attacker has the privilege to reach the training phase, one can potentially poison the model and bias the system towards incorrect detection results, e.g., causative attacks where the attacker influences the training data [41]. In theory, our detection approach is vulnerable to this attack due to the use of a stochastic language. However, our approach is not designed to be an online machine learning system-the model is not constantly updated-and it avoids the problem to some extent. In general, data sanitization provides a more precise model and reduce false negatives for any learning system. Moreover, exploration in adversarial machine learning [26] can further enable the development of online learning algorithms for program anomaly detection in the future.

\section{RELATED WORK}

Conventional program anomaly detection systems (a.k.a., host-based intrusion detection systems) follow Denning's intrusion detection vision [8]. They were designed to detect illegal control flows or anomalous system calls based on two basic paradigms: (i) $n$-gram short call sequence validation that was introduced by Forrest et al. [12]; and (ii) automaton transition verification, which was first described by Kosoresow and Hofmeyr [34] (DFA) and formalized by Sekar et al. [49] (FSA) and Wagner and Dean [59] (NDPDA).

Each path leads to a fruitful line of models for detecting anomalous program behaviors. The basic $n$-gram model was further studied in Inoue and Somayaji [28] and Forrest et al. [11] and sophisticated forms of it were developed, e.g., machine learning models [29, 36], first-order Markov models [64, 65], hidden Markov models [15, 61], and neural network models [17]. Call arguments were used to precisely define states in Maggi et al. [38]. $n$-gram frequencies were studied in Hubballi et al. [27]. Beyond program anomaly detection, $n$-grams was also used in malware detection Canali et al. [5].

The essence of $n$-gram is to model and analyze local features of program traces with a small $n$. Enlarging $n$ results in exponential convergence and storage issues [11]. However, small $n$ (local feature analysis) makes it possible for attackers to evade the detection by constructing a malicious trace, of which any small fragment is normal. Wagner and Soto first demonstrated such a mimicry attack with a malicious sequence of system calls diluted to normal [60].

The other path, i.e., automaton detection, aims to model a program not restricted to short call sequence analysis. It builds an automaton or a pushdown automaton to read the entire trace at a time. However, reading the entire trace is not equivalent to correlating events in the transition history. All automaton models in literature are first-order and they only verify each state transition on its own. Program counter and call stack information were used to help precisely define each state, e.g., a system call, in an automaton $[9,10,49]$. Static analysis was used [59, 63] and Pushdown automaton or its equivalents were employed in many advanced models [9, 19, 30,37]. Hidden procedure transition information is revealed by inserting flags in particular procedures [19]. Call arguments were added in FSA models [18] to improve modeling accuracy, and individual transition frequencies had been analyzed to detect DoS attacks [14].

Some of the existing detection methods are context sensitive $[9,10,19,20,51,62]$, but they are not context-sensitive language models. These models use calling context information to help identify a program state, e.g., a system call. It is different from event co-occurrence analysis because the call 
stack only provides details at a stack snapshot. It is not designed to record all historical execution paths. Events are forgotten when their associated calls are popped from the stack, and they cannot be observed by later events. Event co-occurrence analysis is also not equivalent to path sensitivity since it does not require the analysis of the order of events.

The relation among events that occur far away has not been systematically studied in the literature. The missing analysis allows aberrant path attacks to take place. In this article, we formalize the problem of event correlation analysis within long program traces using a stochastic mildly context-sensitive language and bring forward a detection system that correlates events in long trace segments for event co-occurrence analysis and occurrence frequency analysis. Frequency analysis has been made as extensions to existing models [14,27], but they are restricted by the underlying automaton $/ n$-gram models (regular or context-free languages).

Clustering and classification techniques have been widely used in malware classification $[1,13$, 32, 46]. Malware classification aims at extracting abstract malware behavior signatures and identifies a piece of malware using one or multiple signatures. However, program anomaly detection models normal behaviors and examines an entire profile to decide whether it is normal. It is not sufficient to conclude an incoming behavior is normal because one feature of it is normal.

Correlation analysis techniques were developed to detect network intrusions. Valeur et al. described a comprehensive framework to correlate alerts from various IDS systems [58]. Perdisci et al. proposed $2_{v}$-gram scheme to discover related bytes $v$ positions apart in traffic payload [44, 45]. Correlation analysis, including self-correlation, was used for detecting synchronized and repetitive bot activities [21]. Zhang et al. proposed a system to infer network traffic correlation using pre-defined rules [66] and machine learning methods [67]. These techniques were developed under specific bot behavior hypotheses, e.g., temporal relations of network events. In comparison, we address the program anomaly detection problem by developing new algorithms to overcome the unique behavior diversity and scalability challenges.

Defenses against specific known program attacks have been investigated besides anomaly detection. For example, Moore et al. introduced backscatter analysis to discover DoS attacks [40], and Brumley et al. invented RICH to prevent integer overflow [4]. These defenses target specific attack signatures and cannot detect unknown attacks. Therefore, they are different from general anomaly detection approaches.

\section{CONCLUSIONS AND FUTURE WORK}

In this article, we studied aberrant path attacks, formalized a stochastic mildly context-sensitive language anomaly detection model, and presented a two-stage data mining approach to detect these attacks in long program traces. Our work points out the need for a context-sensitive language level model in response to the development of modern attacks where attackers alter execution paths but not the control flows. Our work demonstrates the effectiveness of large-scale program behavior modeling via event correlation analysis in long program traces and sheds light on the future development of comprehensive context-sensitive language detection model. In future work, we plan to adopt advanced dynamic tracing techniques and build real-time security incidence response systems to enforce program execution security on top of our detection solution.

\section{REFERENCES}

[1] Ulrich Bayer, Paolo Milani Comparetti, Clemens Hlauschek, Christopher Krügel, and Engin Kirda. 2009. Scalable, behavior-based malware clustering. In Proceedings of the Network and Distributed System Security Symposium. The Internet Society, Reston, VA, 8-11.

[2] Johan Behrenfeldt. 2009. A Linguist's Survey of Pumping Lemmata. Master's thesis. University of Gothenburg, Sweden.

[3] S. Bhatkar, A. Chaturvedi, and R. Sekar. 2006. Dataflow anomaly detection. In Proceedings of the IEEE Symposium on Security and Privacy. IEEE Computer Society, Washington, DC, 15-62. 
[4] David Brumley, Dawn Xiaodong Song, Tzicker Chiueh, Rob Johnson, and Huijia Lin. 2007. RICH: Automatically protecting against integer-based vulnerabilities. In Proceedings of the Network and Distributed System Security Symposium. The Internet Society, Reston, VA, 1-28.

[5] Davide Canali, Andrea Lanzi, Davide Balzarotti, Christopher Kruegel, Mihai Christodorescu, and Engin Kirda. 2012. A quantitative study of accuracy in system call-based malware detection. In Proceedings of the 2012 International Symposium on Software Testing and Analysis. ACM, New York, NY, 122-132.

[6] Shuo Chen, Jun Xu, Emre C. Sezer, Prachi Gauriar, and Ravishankar K. Iyer. 2005. Non-control-data attacks are realistic threats. In Proceedings of the USENIX Security Symposium, Vol. 14. USENIX Association, Berkeley, CA, 12-12.

[7] Marco Cova, Davide Balzarotti, Viktoria Felmetsger, and Giovanni Vigna. 2007. Swaddler: An approach for the anomaly-based detection of state violations in web applications. In Proceedings of the International Symposium on Research in Attacks, Intrusions and Defenses. Springer, Berlin, Germany, 63-86.

[8] Dorothy E. Denning. 1987. An intrusion-detection model. IEEE Transactions on Software Engineering 13, 2 (1987), 222-232.

[9] Henry Hanping Feng, Jonathon T. Giffin, Yong Huang, Somesh Jha, Wenke Lee, and Barton P. Miller. 2004. Formalizing sensitivity in static analysis for intrusion detection. In Proceedings of the IEEE Symposium on Security and Privacy. IEEE Computer Society, Washington, DC, 194-208.

[10] Henry Hanping Feng, Oleg M. Kolesnikov, Prahlad Fogla, Wenke Lee, and Weibo Gong. 2003. Anomaly detection using call stack information. In Proceedings of the IEEE Symposium on Security and Privacy. IEEE Computer Society, Washington, DC, 62-75.

[11] Stephanie Forrest, Steven Hofmeyr, and Anil Somayaji. 2008. The evolution of system-call monitoring. In Proceedings of the Annual Computer Security Applications Conference. IEEE Computer Society, Washington, DC, 418-430.

[12] Stephanie Forrest, Steven A. Hofmeyr, Anil Somayaji, and Thomas A. Longstaff. 1996. A sense of self for Unix processes. In Proceedings of the IEEE Symposium on Security and Privacy. IEEE Computer Society, Washington, DC, 120128.

[13] Matt Fredrikson, Somesh Jha, Mihai Christodorescu, Reiner Sailer, and Xifeng Yan. 2010. Synthesizing near-optimal malware specifications from suspicious behaviors. In Proceedings of the IEEE Symposium on Security and Privacy. IEEE Computer Society, Washington, DC, 45-60.

[14] Alessandro Frossi, Federico Maggi, Gian Luigi Rizzo, and Stefano Zanero. 2009. Selecting and improving system call models for anomaly detection. In Detection of Intrusions and Malware, and Vulnerability Assessment. Springer, Hamburg, Germany, 206-223.

[15] Debin Gao, Michael K. Reiter, and Dawn Song. 2006. Behavioral distance measurement using hidden Markov models. In Proceedings of the International Symposium on Research in Attacks, Intrusions and Defenses. Springer, Hamburg, Germany, 19-40.

[16] Xinyang Ge, Weidong Cui, and Trent Jaeger. 2017. GRIFFIN: Guarding control flows using intel processor trace. In Proceedings of the Twenty-Second International Conference on Architectural Support for Programming Languages and Operating Systems (ASPLOS'17). ACM, New York, NY, 585-598.

[17] Anup K. Ghosh, James Wanken, and Frank Charron. 1998. Detecting anomalous and unknown intrusions against programs. In Proceedings of the 14th Annual Computer Security Applications Conference. IEEE Computer Society, Washington, DC, 259-267.

[18] Jonathon T. Giffin, David Dagon, Somesh Jha, Wenke Lee, and Barton P. Miller. 2006. Environment-sensitive intrusion detection. In Proceedings of the International Symposium on Research in Attacks, Intrusions and Defenses. Springer, Hamburg, Germany, 185-206.

[19] Jonathon T. Giffin, Somesh Jha, and Barton P. Miller. 2004. Efficient context-sensitive intrusion detection. In Proceedings of the Network and Distributed System Security Symposium. The Internet Society, Reston, VA, 0.

[20] Rajeev Gopalakrishna, Eugene H. Spafford, and Jan Vitek. 2005. Efficient intrusion detection using automaton inlining. In Proceedings of the IEEE Symposium on Security and Privacy. IEEE Computer Society, Washington, DC, 18-31.

[21] Guofei Gu, Phillip A. Porras, Vinod Yegneswaran, Martin W. Fong, and Wenke Lee. 2007. BotHunter: Detecting malware infection through IDS-driven dialog correlation. In Proceedings of the USENIX Security Symposium, Vol. 7. USENIX Association, Berkeley, CA, 1-16.

[22] Zhongshu Gu, Kexin Pei, Qifan Wang, Luo Si, Xiangyu Zhang, and Dongyan Xu. 2014. LEAPS: Detecting camouflaged attacks with statistical learning guided by program analysis. In Proceedings of the Annual IEEE/IFIP International Conference on Dependable Systems and Networks. IEEE Computer Society, Washington, DC, 491-502.

[23] Heartbleed 2014. The Heartbleed Bug. Retrieved from http://heartbleed.com/.

[24] Hong Hu, Zheng Leong Chua, Sendroiu Adrian, Prateek Saxena, and Zhenkai Liang. 2015. Automatic generation of data-oriented exploits. In Proceedings of the 24th USENIX Conference on Security Symposium. USENIX Association, Berkeley, CA, 177-192. 
[25] Hong Hu, Shweta Shinde, Sendroiu Adrian, Zheng Leong Chua, Prateek Saxena, and Zhenkai Liang. 2016. Dataoriented programming: On the expressiveness of non-control data attacks. In Proceedings of the IEEE Symposium on Security and Privacy. IEEE Computer Society, Washington, DC, 969-986.

[26] Ling Huang, Anthony D. Joseph, Blaine Nelson, Benjamin I. P. Rubinstein, and J. D. Tygar. 2011. Adversarial machine learning. In Proceedings of the 4th ACM Workshop on Security and Artificial Intelligence. ACM, New York, NY, 43-58.

[27] N. Hubballi, S. Biswas, and S. Nandi. 2011. Sequencegram: N-gram modeling of system calls for program based anomaly detection. In Proceedings of the International Conference on Communication Systems and Networks. IEEE, Washington, DC, 1-10.

[28] Hajime Inoue and Anil Somayaji. 2007. Lookahead pairs and full sequences: A tale of two anomaly detection methods. In Proceedings of the Annual Symposium on Information Assurance. ASIA, Albany, NY, 9-19.

[29] Md Rafiqul Islam, Md Saiful Islam, and Morshed U. Chowdhury. 2011. Detecting unknown anomalous program behavior using API system calls. In Informatics Engineering and Information Science. Springer, Hamburg, Germany, 383-394.

[30] Jafar Haadi Jafarian, Ali Abbasi, and Siavash Safaei Sheikhabadi. 2011. A gray-box DPDA-based intrusion detection technique using system-call monitoring. In Proceedings of the Annual Collaboration, Electronic Messaging, Anti-Abuse and Spam Conference. ACM, New York, NY, 1-12.

[31] Rohit Jalan and Arun Kejariwal. 2012. Trin-Trin: Who's calling? A pin-based dynamic call graph extraction framework. International fournal of Parallel Programming 40, 4 (2012), 410-442.

[32] Sandeep Karanth, Srivatsan Laxman, Prasad Naldurg, Ramarathnam Venkatesan, J. Lambert, and Jinwook Shin. 2010. Pattern Mining for Future Attacks. Technical Report MSR-TR-2010-100. Microsoft Research.

[33] Baris Kasikci, Benjamin Schubert, Cristiano Pereira, Gilles Pokam, and George Candea. 2015. Failure sketching: A technique for automated root cause diagnosis of in-production failures. In Proceedings of the 25th Symposium on Operating Systems Principles (SOSP'15). ACM, New York, NY, 344-360.

[34] Andrew P. Kosoresow and Steven A. Hofmeyr. 1997. Intrusion detection via system call traces. IEEE Software 14, 5 (1997), 35-42.

[35] Jeffrey P. Lanza. 2001. SSH CRC32 attack detection code contains remote integer overflow. (2001). Vulnerability Notes Database.

[36] Wenke Lee and Salvatore J. Stolfo. 1998. Data mining approaches for intrusion detection. In Proceedings of the USENIX Security Symposium. USENIX Association, Berkeley, CA, 6-6.

[37] Zhen Liu, Susan M. Bridges, and Rayford B. Vaughn. 2005. Combining static analysis and dynamic learning to build accurate intrusion detection models. In Proceedings of IEEE International Workshop on Information Assurance. IEEE Computer Society, Washington, DC, 164-177.

[38] Federico Maggi, Matteo Matteucci, and Stefano Zanero. 2010. Detecting intrusions through system call sequence and argument analysis. IEEE Transactions on Dependable and Secure Computing 7, 4 (2010), 381-395.

[39] J. Sukarno Mertoguno. 2014. Human decision making model for autonomic cyber systems. International fournal on Artificial Intelligence Tools 23, 06 (2014), 1460023.

[40] David Moore, Colleen Shannon, Douglas J. Brown, Geoffrey M. Voelker, and Stefan Savage. 2006. Inferring internet denial-of-service activity. ACM Transactions on Computer Systems 24, 2 (2006), 115-139.

[41] James Newsome, Brad Karp, and Dawn Song. 2006. Paragraph: Thwarting signature learning by training maliciously. In Proceedings of the International Symposium on Research in Attacks, Intrusions and Defenses (RAID). Springer, Berlin, Germany, 81-105.

[42] Paradyn 2016. The Paradyn Project. Retrieved from http://www.paradyn.org/.

[43] K. Peason. 1901. On lines and planes of closest fit to systems of point in space. Philos. Mag. 2 (1901), 559-572.

[44] Roberto Perdisci, Davide Ariu, Prahlad Fogla, Giorgio Giacinto, and Wenke Lee. 2009. McPAD: A multiple classifier system for accurate payload-based anomaly detection. Computer Networks 53, 6 (2009), 864-881.

[45] R. Perdisci, Guofei Gu, and Wenke Lee. 2006. Using an ensemble of one-class SVM classifiers to harden payload-based anomaly detection systems. In Proceedings of the International Conference on Data Mining. IEEE Computer Society, Washington, DC, 488-498.

[46] Roberto Perdisci, Wenke Lee, and Nick Feamster. 2010. Behavioral clustering of HTTP-based malware and signature generation using malicious network traces. In Proceedings of the USENIX Symposium on Networked Systems Design and Implementation. USENIX Association, Berkeley, CA, 26-26.

[47] Geoffrey K. Pullum. 1983. Context-freeness and the computer processing of human languages. In Proceedings of the Annual Meeting on Association for Computational Linguistics. ACL, Stroudsburg, PA, 1-6.

[48] Bernhard Schölkopf, Robert C. Williamson, Alex J. Smola, John Shawe-Taylor, and John C. Platt. 1999. Support vector method for novelty detection. In Proceedings of the Annual Conference on Neural Information Processing Systems, Vol. 12. The MIT Press, Cambridge, MA, 582-588. 
[49] R. Sekar, Mugdha Bendre, Dinakar Dhurjati, and Pradeep Bollineni. 2001. A fast automaton-based method for detecting anomalous program behaviors. In Proceedings of the IEEE Symposium on Security and Privacy. IEEE Computer Society, Washington, DC, 144-155.

[50] Hovav Shacham, Matthew Page, Ben Pfaff, Eu-Jin Goh, Nagendra Modadugu, and Dan Boneh. 2004. On the effectiveness of address-space randomization. In Proceedings of the ACM Conference on Computer and Communications Security. ACM, New York, NY, 298-307.

[51] Monirul Sharif, Kapil Singh, Jonathon Giffin, and Wenke Lee. 2007. Understanding precision in host based intrusion detection. In Proceedings of the International Symposium on Research in Attacks, Intrusions and Defenses. Springer, Hamburg, Germany, 21-41.

[52] Xiaokui Shu and Danfeng Yao. 2016. Program anomaly detection: Methodology and practices. In Proceedings of the 2016 ACM SIGSAC Conference on Computer and Communications Security (CCS'16). ACM, New York, NY, 1853-1854.

[53] Xiaokui Shu, Danfeng Yao, and Naren Ramakrishnan. 2015. Unearthing stealthy program attacks buried in extremely long execution paths. In Proceedings of the 2015 ACM Conference on Computer and Communications Security (CCS). ACM, New York, NY, 401-413.

[54] Xiaokui Shu, Danfeng Yao, and Barbara G. Ryder. 2015. A formal framework for program anomaly detection. In Proceedings of the 18th International Symposium on Research in Attacks, Intrusions and Defenses (RAID). Springer, Hamburg, Germany, 270-292.

[55] Alexander Sotirov. 2007. Heap Feng Shui in JavaScript. (2007). Black Hat Europe.

[56] S. C. Sundaramurthy, J. McHugh, X. S. Ou, S.R. Rajagopalan, and M. Wesch. 2014. An anthropological approach to studying CSIRTs. IEEE Security \& Privacy 12, 5 (September 2014), 52-60.

[57] Systemtap. 2006. SystemTap Overhead Test, https://sourceware.org/ml/systemtap/2006-q3/msg00146.html. (2006).

[58] Fredrik Valeur, Giovanni Vigna, Christopher Kruegel, and Richard A. Kemmerer. 2004. A comprehensive approach to intrusion detection alert correlation. IEEE Transactions on Dependable and Secure Computing 1, 3 (July 2004), 146-169.

[59] David Wagner and R. Dean. 2001. Intrusion detection via static analysis. In Proceedings of the IEEE Symposium on Security and Privacy. IEEE Computer Society, Washington, DC, 156-168.

[60] David Wagner and Paolo Soto. 2002. Mimicry attacks on host-based intrusion detection systems. In Proceedings of the ACM Conference on Computer and Communications Security. ACM, New York, NY, 255-264.

[61] Christina Warrender, Stephanie Forrest, and Barak Pearlmutter. 1999. Detecting intrusions using system calls: Alternative data models. In Proceedings of the IEEE Symposium on Security and Privacy. IEEE Computer Society, Washington, DC, 133-145.

[62] K. Xu, K. Tian, D. Yao, and B. G. Ryder. 2016. A sharper sense of self: Probabilistic Reasoning of program behaviors for anomaly detection with context sensitivity. In Proceedings of the 46th Annual IEEE/IFIP International Conference on Dependable Systems and Networks (DSN). IEEE, Washington, DC, 467-478.

[63] K. Xu, D. D. Yao, B. G. Ryder, and K. Tian. 2015. Probabilistic program modeling for high-precision anomaly classification. In Proceedings of the IEEE 28th Computer Security Foundations Symposium. IEEE, Washington, DC, 497-511.

[64] Nong Ye and X. Li. 2000. A Markov chain model of temporal behavior for anomaly detection. In Proceedings of the 2000 IEEE Systems, Man, and Cybernetics Information Assurance and Security Workshop, Vol. 166. IEEE, Washington, DC, 169.

[65] Stefano Zanero. 2004. Behavioral intrusion detection. In Computer and Information Sciences. Springer, Hamburg, Germany, 657-666.

[66] Hao Zhang, Danfeng Yao, and Naren Ramakrishnan. 2014. Detection of stealthy malware activities with traffic causality and scalable triggering relation discovery. In Proceedings of the 9th ACM Symposium on Information, Computer and Communications Security. ACM, New York, NY, 39-50.

[67] Hao Zhang, Danfeng Yao, Naren Ramakrishnan, and Zhibin Zhang. 2016. Causality reasoning about network events for detecting stealthy malware activities. Computers \& Security 58 (2016), 180-198.

Received July 2016; revised February 2017; accepted May 2017 